\title{
溶液酸碱度调控的分子机器与分子开关
}

\author{
翁官欢*, $a, b$ 朱 涁 $b$ 叶 杨 $b$ 李世军*,b \\ $\left({ }^{a}\right.$ 台州学院医药化工学院 台州 318000) \\ ( ${ }^{b}$ 杭州师范大学材料与化学化工学院 杭州 310036)
}

\begin{abstract}
摘要 人工分子机器按其能量驱动方式可以分为化学驱动、电化学驱动和光驱动三类. 溶液酸碱度调控( $\mathrm{pH}$ 调控)驱动 属于化学驱动的一种, 其基本原理是通过加入酸碱性化合物调节溶液的酸碱度从而改变体系中各种组分的化学性质, 实现分子机器的运动. 利用溶液酸碱度调控已成为人工组装分子机器最常用的能量供应方式之一. 对溶液酸碱度调控 的分子机器和分子开关进行了详细综述, 分别总结了基于冠醚和其他大环主体的溶液酸碱度调控分子机器与分子开关 的制备及酸碱调控方法, 并展望了它们在信息存储、电子器件及药物传递等领域中的应用.
\end{abstract}

关键词 分子机器; 分子识别; 冠醚; 穴状配体; 主客体化合物; 轮烷; 超分子化学; 分子器件

\section{Acid/Base-Controllable Molecular Machines and Molecular Switches}

\author{
Weng, Guan-Huan*,a,b \\ Zhu, $\operatorname{Bin}^{b}$ \\ Ye, Yang ${ }^{b}$ \\ Li, Shijun*,b \\ ( ${ }^{a}$ College of Pharmaceutical and Chemical Engineering, Taizhou University, Taizhou 318000) \\ ( ${ }^{b}$ College of Material, Chemistry and Chemical Engineering, Hangzhou Normal University, Hangzhou 310036)
}

\begin{abstract}
There are three kinds of energy-supplied modes to drive artificial molecular machines: chemical stimulation, electrochemical input and light stimulation. The basic principle of acid/base-controlled (or $\mathrm{pH}$-operated) method is to change the chemical properties of components in the system by adjusting $\mathrm{pH}$ of the solution via adding acid/base compounds, to realize the movements of components, which belongs to chemical stimulation mode. The acid/base controlled method has been one of the most useful energy-driven modes for artificial molecular machines. This article summarizes the recent studies on acid/basecontrollable molecular machines or molecular switches. The synthesis and operation of acid/base-controllable artificial molecular switches and molecular machines based on crown ethers and other macrocyclic hosts are reviewed. They exhibited great potential in the applications for information storage materials, electronic devices, and drug delivery.
\end{abstract}

Keywords molecular machines; molecular recognition; crown ethers; cryptands; host-guest compounds; rotaxanes; supramolecular chemistry; molecular devices

随着社会进步和科技创新, 各种宏观机器正朝着更 小化发展, 纳米世界充满着无穷的魅力. 但是传统的由 大到小的物理方法已越来越显示出其制备尺寸的局限 性, 而由小至大的化学方法为纳米材料和分子尺度材料 的制备提供了一种高效的方法. 化学家们正试图通过由 小至大的方法探索纳米的世界, 实现利用单分子或者超 分子组装来模拟并最终替代宏观世界中的许多过程, 从 而实现器件的纳米尺寸化 ${ }^{[1]}$. 分子机器, 即指在分子水 平上发生可控运动的机器, 正是这样一种将能量转变为
可控运动的分子尺度的器件. 按其驱动方式, 分子机器 可以分为化学驱动、电化学驱动和光驱动三类 ${ }^{[1]}$. 溶液 酸碱度调控( $\mathrm{pH}$ 调控)驱动属于化学驱动的一种, 其基本 原理是通过加入酸碱性化合物调节溶液的酸碱度从而 改变体系中各种组分的化学性质，导致结构发生变化并 使组分间进行相对移动, 从而实现分子机器运动 ${ }^{[2]}$. 由 于其操作的简便性及广泛的应用前景, 利用溶液酸碱度 调控已成为人工组装分子机器最常用的能量供应方式 之一.

*E-mail: 1_shijun@hznu.edu.cn; wengguanhuan@126.com

Received September 9, 2014; revised October 24, 2014; published online November 13, 2014.

Project supported by the National Natural Science Foundation of China (Nos. 21072039, 91127010), the Program for Changjiang Scholars and Innovative Research Team in Chinese University (No. IRT1231), and the Zhejiang Provincial Natural Science Foundation of China (No. LZ13B030001).

国家自然科学基金(Nos. 21072039, 91127010)、教育部创新团队(No. IRT1231)、浙江省自然科学基金(No. LZ13B030001)资助项目. 


\section{1 基于冠醚的酸碱调控分子机器与分子开关}

相同的主体在识别不同的客体时, 存在着一定的差 异 ${ }^{[3]}$ (即识别竞争). 基于这个特点, Stoddart 小组 ${ }^{[4]}$ 制备 了具有荧光和氧化还原活性封端单元的二站点分子机 器(Eq. 1). 在该[2]轮烷中, 客体上的两个不同识别位点 表明了可能存在着两种构型, 即双苯并 24-冠-8 (DB24C8)环绕在二级胺盐或 4,4'-联吡啶的识别位点中 心. 核磁共振和单晶 $\mathrm{X}$ 衍射证实了 [2]轮烷处于一开始 处于前者的状态, 这是由于 $\mathrm{DB} 24 \mathrm{C} 8$ 与二级胺盐比与 4,4'-联吡啶盐具有更高的络合能力, 占据竞争优势. 而 通过酸碱调节就能够改变并驱使它从一种形式转变到 另一种, 即形成分子机器. 他们还对一侧有葱环苂光封 端的轮烷进行了酸碱驱动的研究. 碱化处理, 二级胺盐 的识别位点被移除, DB24C8 就自发的往 4,4'-联吡啶中 心移动, 酸化后, 它回到初始状态, 继续酸化, DB24C8 自发地离开二级胺盐识别位点. 此过程中, 他们得到了 主体 DB24C8 两个方向上穿梭过程中的活化参数, 发现 氨/胺组件间的去质子化/再质子化存在着差异以致于主
体的正向和逆向穿梭存在不一致性. 这种具有双稳态特 性的体系为实现分子开关奠定了基础.

Ashton 和 Stoddart 等 ${ }^{[5]}$ 还研究了基于 4,4'-联吡啶盐 与二级胺盐识别位点的双稳态索烃分子机器, 分别采用 氧化还原和酸碱调控两种方式来驱动索烃分子机器运 动(Scheme 1). 采用氧化还原方法时, 伴随着电子的转 移, 吸收波谱上的峰发生强烈变化, 大环上的苂光位点 也被淬灭. 此类基于单电子还原和酸碱双重调控的过程 展现了 AND 逻辑关系.

为了构建高度有序的复杂超分子结构, 并实现分子 的可控运动, Stoddart 小组 ${ }^{[6]}$ 制备了分子级别的纳米电梯 (Eq. 2). 在三维的机械互锁结构中, 具有三个相同的二 级胺盐和联吡啶支链的刚性单元与包含三个 DB24C 8 结 构的对称主体进行识别, 在乙腈或二氯甲烷中, 以垂直 状态形成准轮烷后，大位阻的 3,5-二叔丁基茮溴进行封 端. 这种带有一个三叉平台式互锁结构的机器仅有 2.5 3.5 nm 大小. 伴随着质子化和去质子化, 主体沿刚 性链做上下往复运动而无法脱落, 成了电梯的动力, 扮 演着“电”的角色. 它表现出了明确的通道可逆行为, 这<smiles>CCc1cc(C(C)(C)C)cc(C(C)(C)C)c1</smiles>
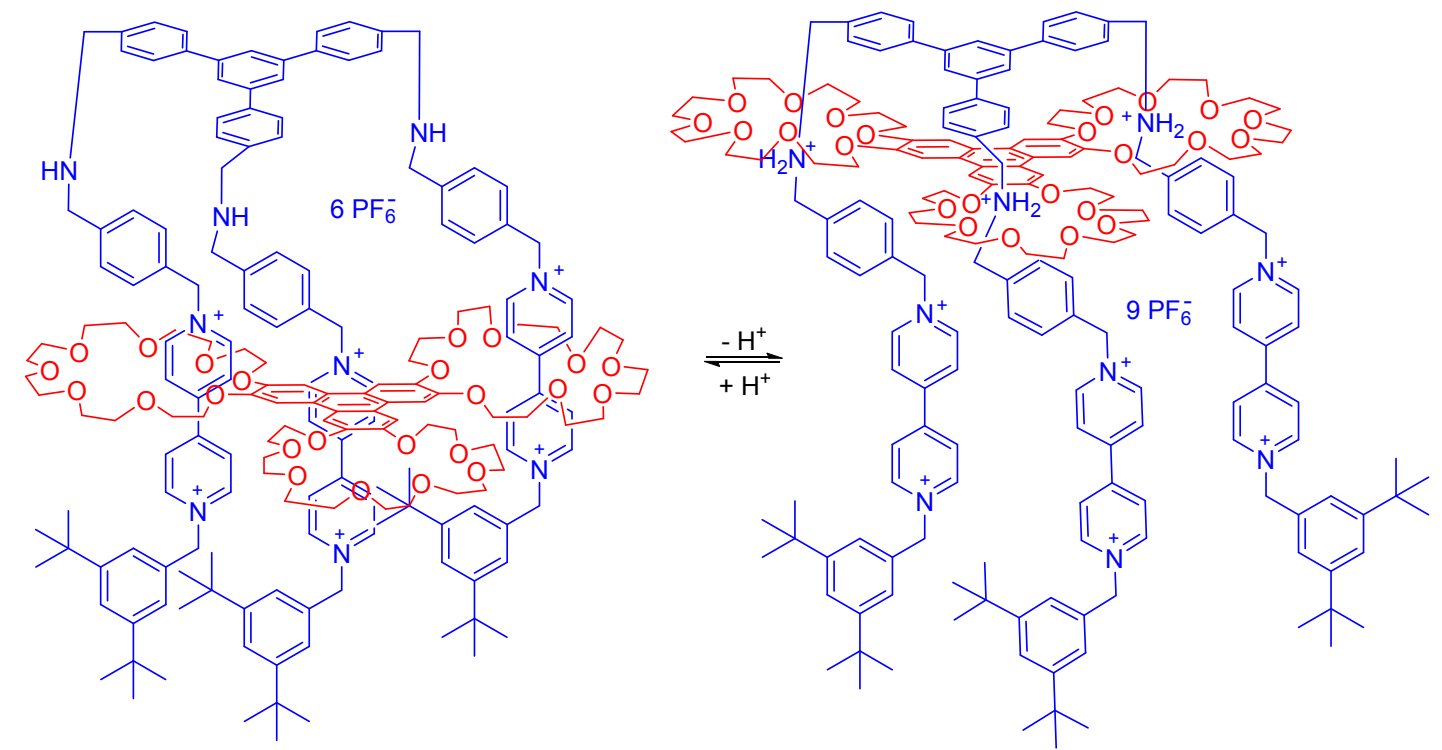


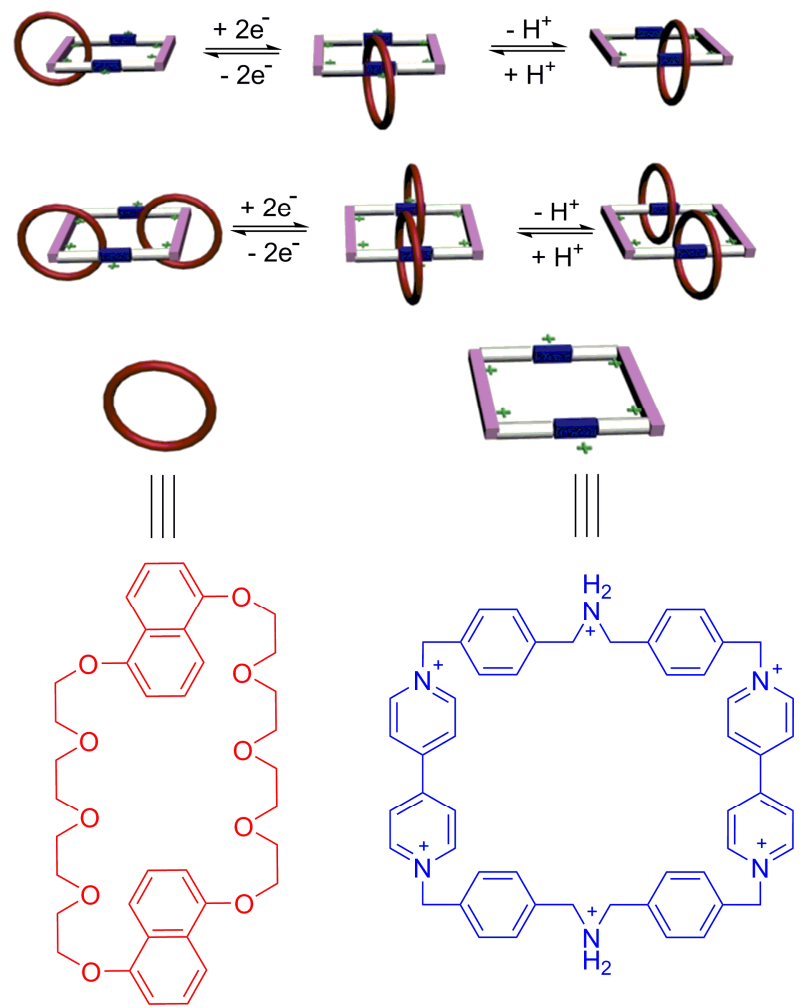

图式 1 氧化还原和酸/碱调控下[2]索烃、[3]索烃运动示意图 Scheme 1 The "co-conformational" switching processes associated with redox stimulation of [2]catenane and [3]catenane

比过去所报道的人工分子机器在结构上更加复杂、易于 组装调控，成为真正的分子水平上的纳米电梯.

Stoddart 小组 ${ }^{[7]}$ 在前期工作基础上, 以 $\mathrm{DB} 24 \mathrm{C} 8$ 衍生 化的 $\mathrm{AB}$ 单体形成二聚体为识别机理, 采用双穿针法, 并利用 3,5-二叔丁基苠澳封端制备了双稳态 [ $c 2$ ]菊花链 化合物(Eq. 3). 这种链能够在酸碱处理后进行可逆的拉 伸和收缩, 形成分子机器. 为了构建可发生酸碱调控运 动的聚合物, 他们在封端基团中引入末端烯(炔), 制备 了功能化的双炔烃 $[c 2]$ 菊花链作为( $\mathrm{AA}$ )单体, 将其与双 叠氮化合物(BB)单体通过点击化学(click)反应聚合, 获 得聚合度约为 11 的线性主链型机械互锁聚合物. 在酸 碱作用下，该机械互锁聚合物发生可控的拉伸和收缩运 动. 最近, Giuseppone 等 $^{[8]}$ 也通过 click 反应制备了基于 DB24C8/二级胺盐识别 A-B 单体的三联吡啶封端的 [c2] 菊花链互锁结构, 再与锌、铁等过渡金属离子配位形成 了机械互锁的配位聚合物, 这种 [ $c 2$ ]菊花链配位聚合物 也可通过酸碱调控实现冠醚主体在二级胺盐与三唑盐 之间的可控移动, 由于其聚合度高达近 3000, 整条链的 收缩和拉伸就如人造肌肉, 真正实现了由分子运动变为 宏观运动. 这些工作为智能可控运动材料的制备开启了 一扇大门.

在不同或者差异较大的识别单元中, 二站点分子机 器中主体更倾向于被固定在强作用识别单元上, 这是由
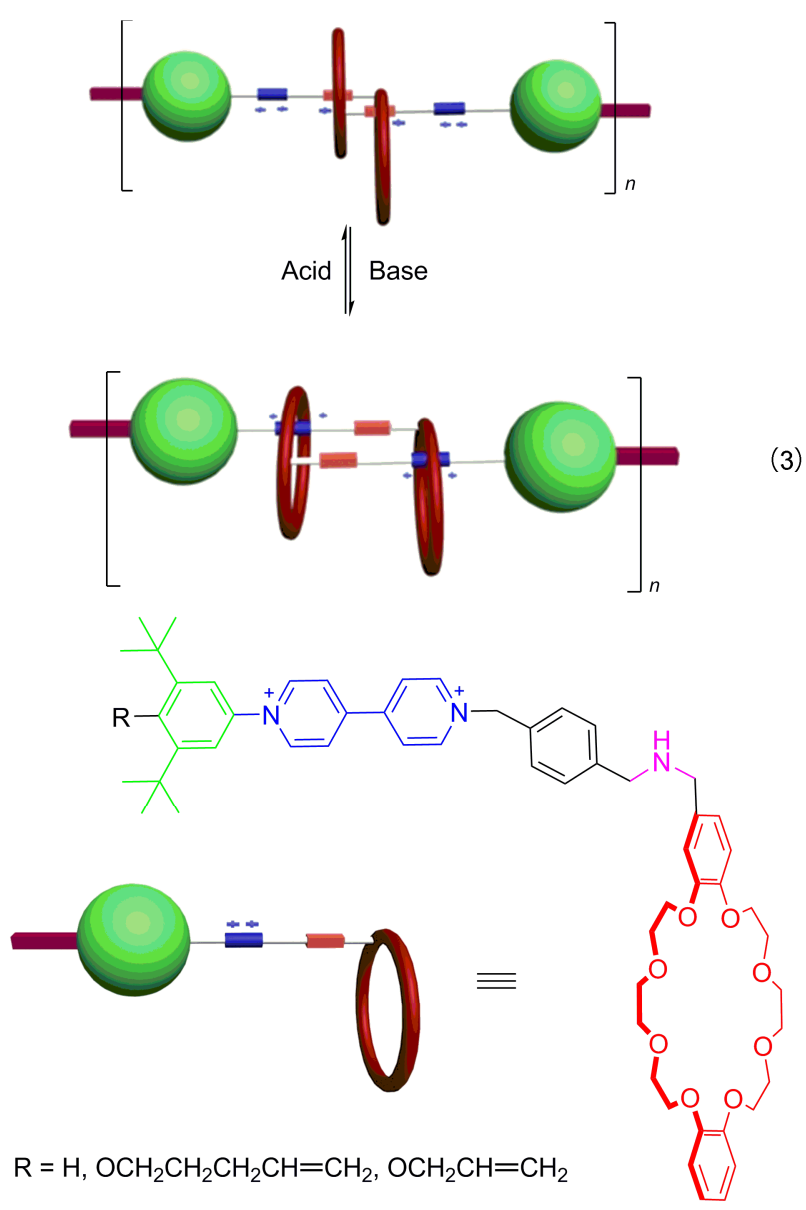

于非共价键作用力的差异造成的. 然而，当相同或相似 的两个识别位点对同一主体进行络合时，会出现什么状 况呢? Leigh 小组 ${ }^{[9]}$ 发现相似的两种识别单元在[2]轮烷 中, 络合主体各占一定的比例(两种物体的比例为 $1: 2$ ). 由于二级胺盐的性质差异，逐滴加入碱可以去除其中的 一个识别位点, 进而实现主体的定向移动, 在这种情况 下, 两种物体在溶液中以 $5: 1$ 的比例存在; 当逐滴滴加 酸, 质子化二级胺, 回到初始状态, 两个识别单元对 DB24C8 又形成竞争(Scheme 2). 这种能够实现酸碱调 控的双稳态结构有望应用到更复杂的纳米器件中.

Coutrot 小组 ${ }^{[10]}$ 采用 $\mathrm{CuAAC}$ 点击化学, 开发了以 DB24C8 为主体、基于 $N$-甲基三坐盐和二级苯胺盐识别 位点的二站点分子机器(Scheme 3). 与识别单元 $N$-甲基 三唑盐相比, 苯胺盐具有更强的络合能力, 因此, 质子 化/去质子化能够调节这种 $\mathrm{pH}$ 敏感的轮烷处于两种不同 的状态.

近来他们 ${ }^{[11]}$ 通过类似的方法开发了基于 [1]轮烷的 分子机器(Eq. 4). 在高度稀释的 $\mathrm{CD}_{2} \mathrm{Cl}_{2}$ 中线性客体自扭 转穿入大环后, 利用三唑的反应特性进行烷基化封端得 到稳定的轮烷, 并产生新的识别位点. 质子化/去质子化 后，大环停留在二级胺盐/三唑盐单元上，整个分子表现 出紧凑/疏松的形式. 这种伸缩运动的分子机器有望于 
有机化学

综述与进展

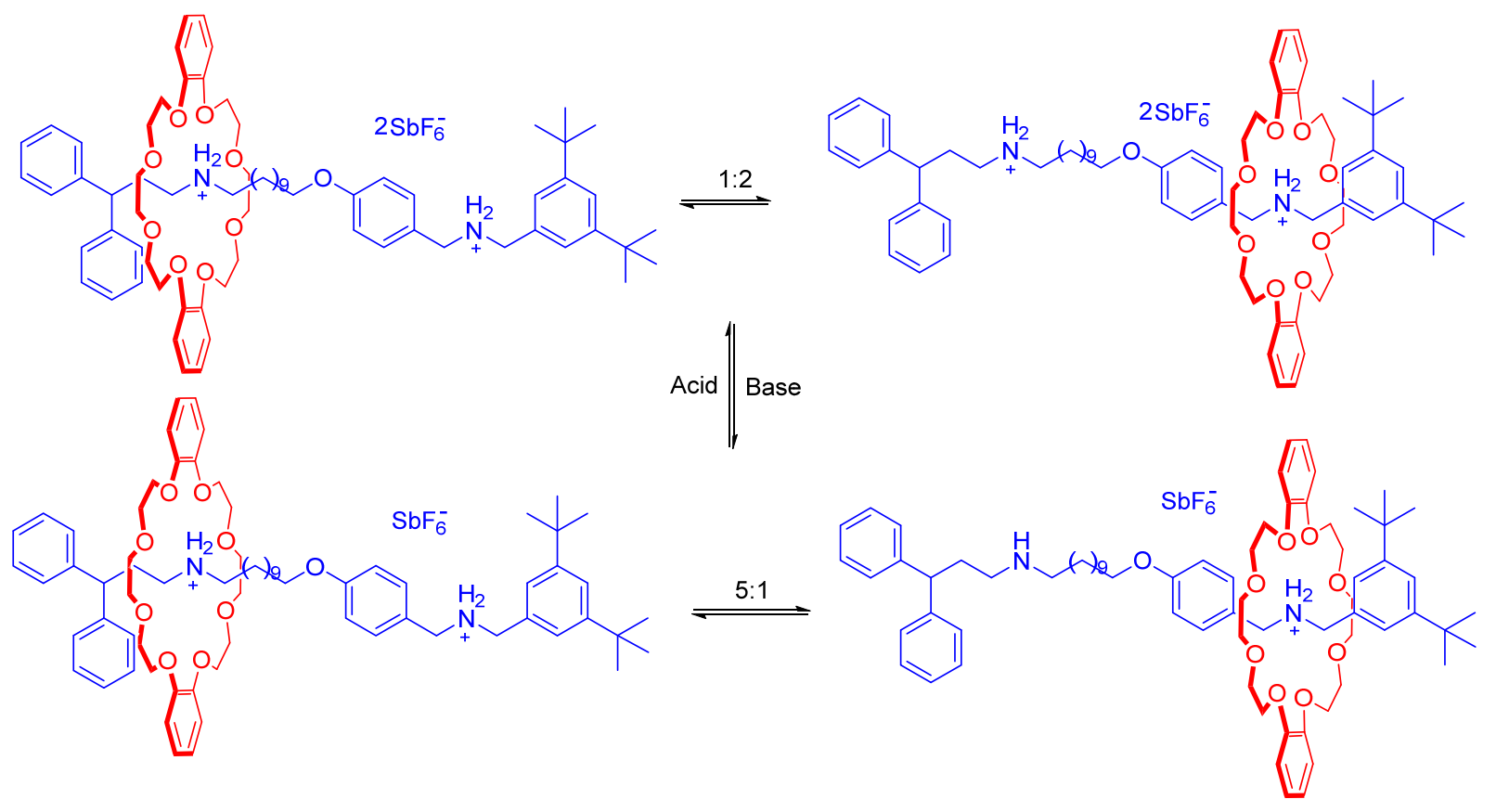

图式 2 在单/双质子化形式下冠醚主体在轮烷中的位置变化

Scheme 2 An ammonium/bis-ammonium switchable molecular machine
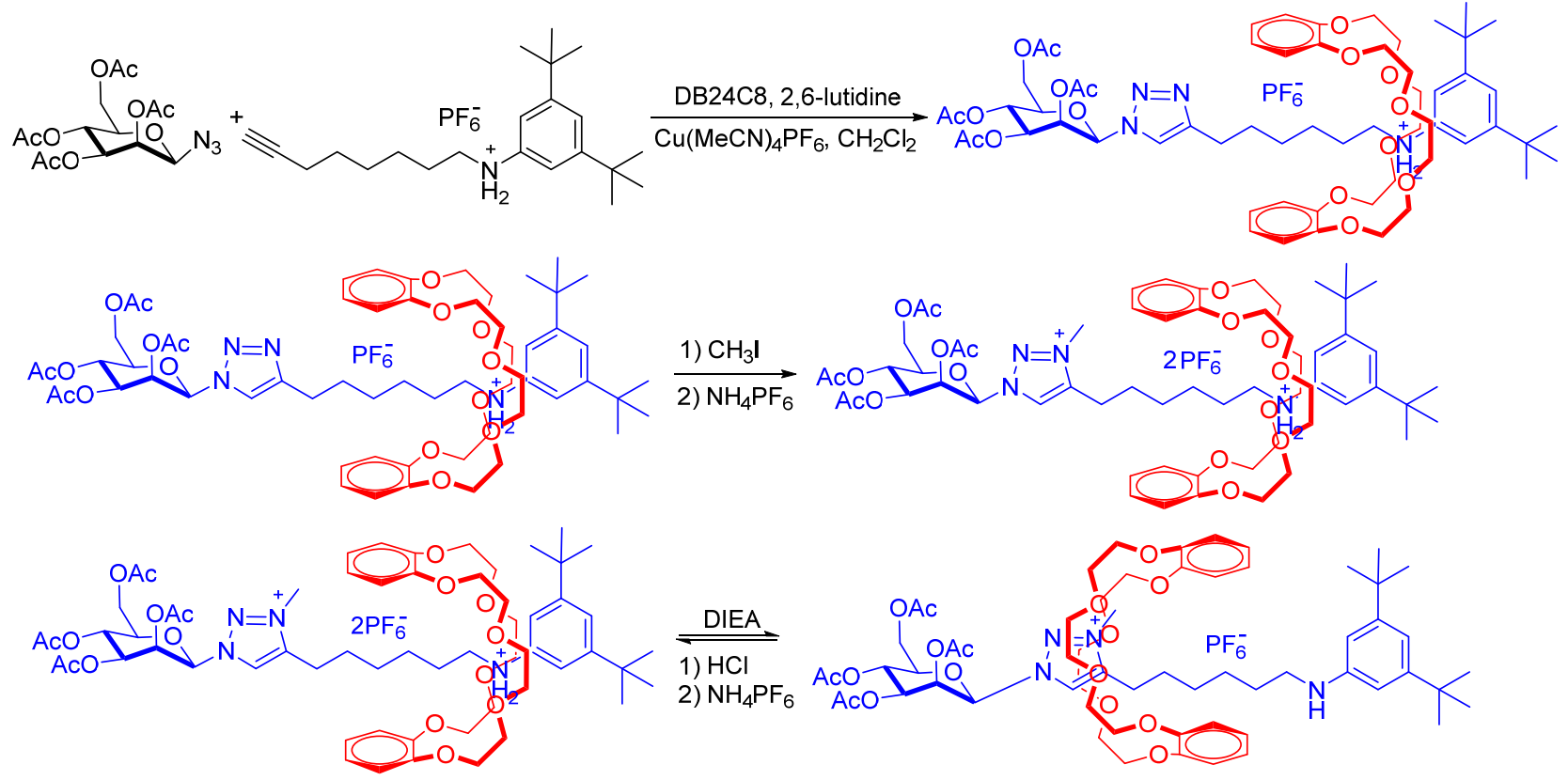

图式 3 基于 $N$-甲基三唑盐和苯胺盐识别位点的二站点酸碱调控分子机器

Scheme 3 A new glycorotaxane molecular machine based on an anilinium and a triazolium station
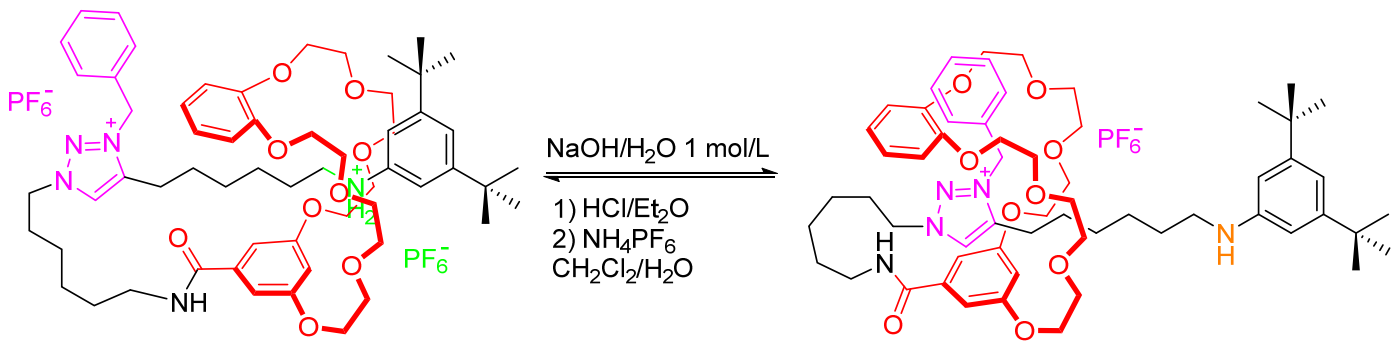

(4)

312

http://sioc-journal.cn/

() 2015 Chinese Chemical Society \& SIOC, CAS

Chin. J. Org. Chem. 2015, 35, 309 324 
应用到生物驱虫剂中。

Credi 和 Stoddart 等 ${ }^{[12]}$ 研究了基于冠醚/DAP 盐准轮 烷识别的溶液酸碱度调控分子梭运动(Eq. 5). 大环主体 BPP34C10 或 DN38C10 和线性电子受体 DAP 盐(2,7-二 芐基二氮杂萠双阳离子) 在溶液中自组装形成准轮烷. 由于 DAP 盐和有机胺形成更强的络合物, 因此, 逐滴滴 加碱液, 主客体发生分离; 加入等量的酸, 主客体重新 络合. 如此循环往复, 实现了溶液酸碱度调控的分子梭. DAP 的苂光在解离/重穿入时能够发生强烈的变化. 这 种投入(化学输入)/产出(苂光性)分子水平特征体系有望 成为一个 XOR 逻辑门.

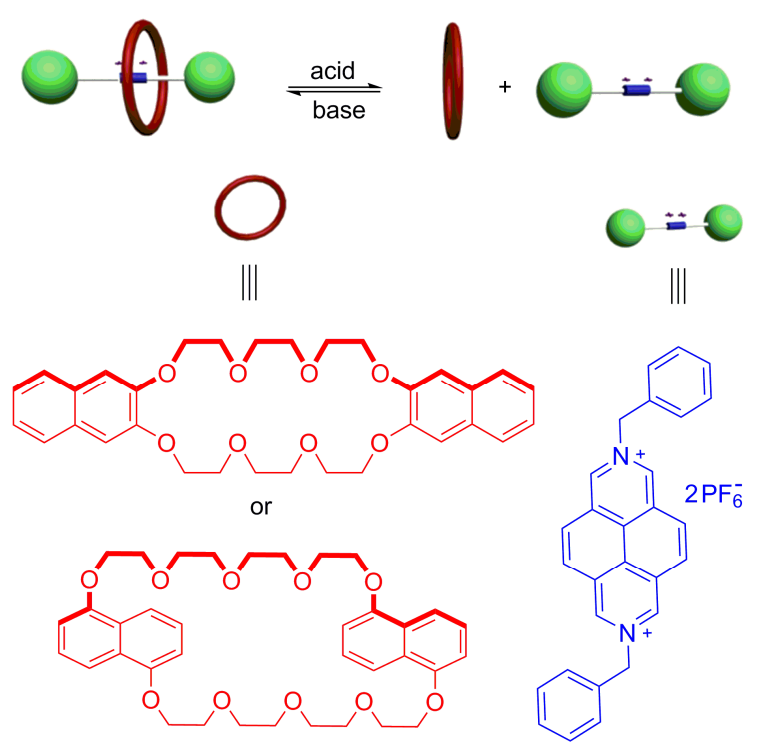

最近, 黄飞鹤小组 ${ }^{[13]}$ 发展了基于 $\mathrm{BMP} 32 \mathrm{C} 10$ 衍生 物和 DAP 盐、邻菲咯啉盐的主客体化学(Eq. 6). 它们之 间形成互穿的准轮烷, 并在晶体结构中 BMP $32 \mathrm{C} 10$ 衍生 物/DAP 盐通过氢键和 $\pi-\pi$ 堆积作用形成了链状的聚准 轮烷结构. 另外, 该准轮烷也可以在二乙胺和三氟乙酸 的作用下发生解离/络合, 实现主客体在溶液中可控识 别.

黄飞鹤小组 ${ }^{[14]}$ 还报道了 $\mathrm{BMP} 32 \mathrm{C} 10$ 的二吡啶衍生 物与乙烯双吡啶盐通过 $\pi-\pi$ 堆积、氢键等作用自组装成 taco 型的夹心络合物. 主体上的两个吡啶基对酸碱刺激 具有响应性, 使主客体络合发生酸碱调控的可逆络合与 解离(Eq. 7).

李玉良小组 ${ }^{[15]}$ 利用 $\mathrm{Cu}$ 催化的 1,3-偶极环加成反应 制备了具有荧光响应的紧凑型[2]轮烷(Eq. 8). 在碱性条 件下, 大环能够自主地从二级胺盐处移动至三唑盐部位 并产生苂光响应; 移除 DIEA 时, 主体回到原始状态, 苂光消失. 整个运动过程伴随着分子内部产生光诱导的 电子转移, 从而改变光学性质, 有望应用到酸碱控制的 苂光响应分子开关.

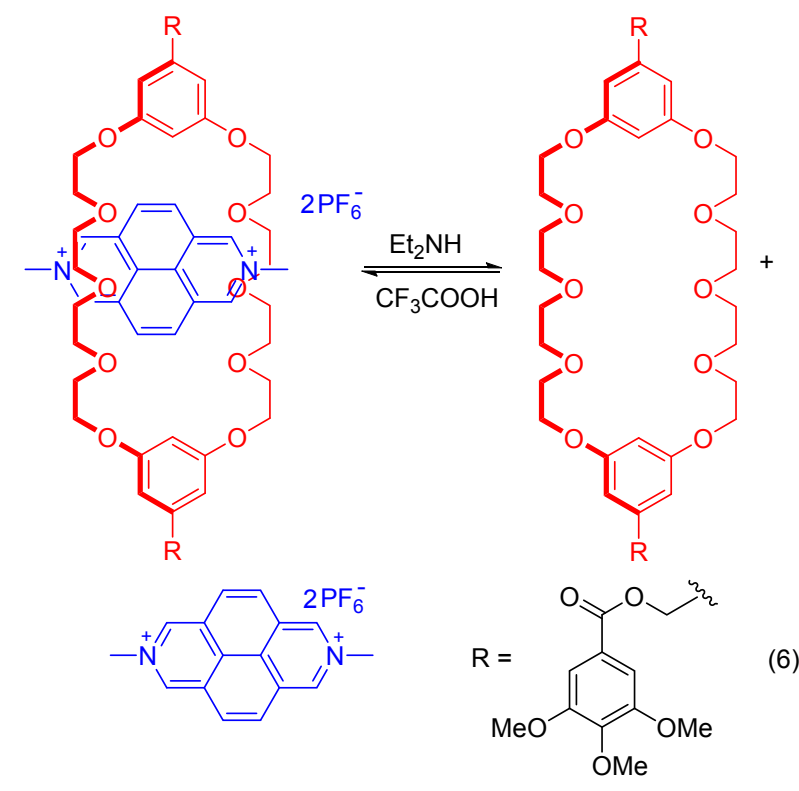

(6)

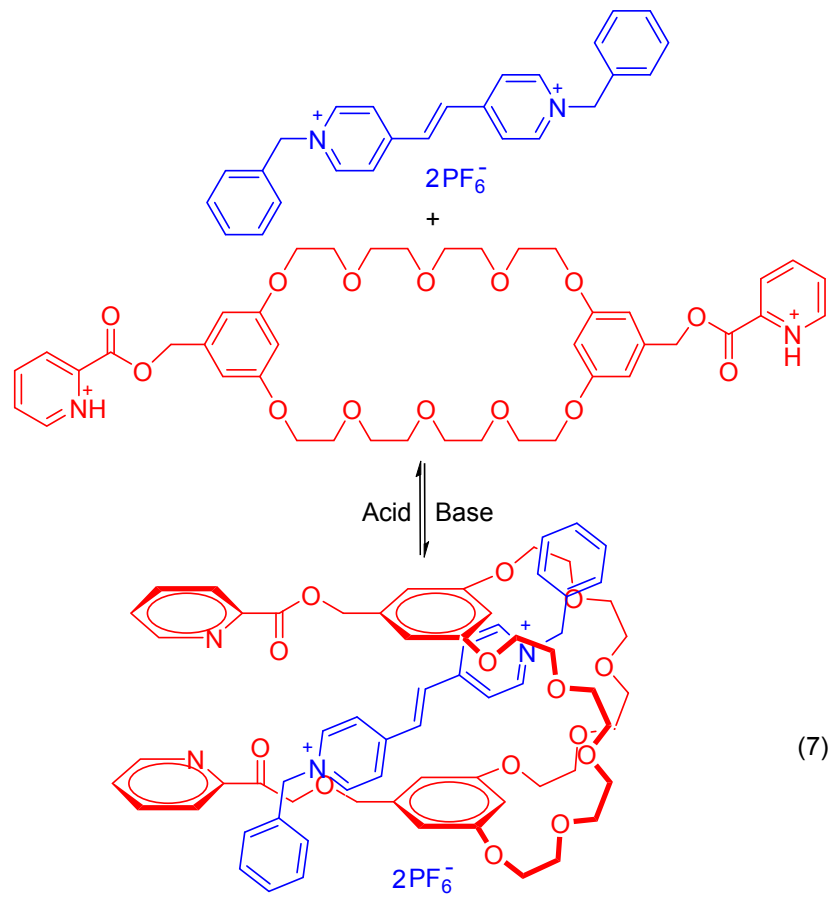

此外，他们 ${ }^{[16]}$ 还制备了基于氢键和 $\pi$ 给体 $/ \pi$ 受体模 板导向的酸碱可控[2]索烃分子机器(Eq. 9). 与其它分子 机器不同的是，除了存在主客体之间的识别，主体上的 菜基与客体上的大环还具有 $\pi-\pi$ 堆积和电荷转移作用, 主体是氢键受体的同时也在扮演着 $\pi$ 给体的双重角色. 在质子化/去质子化过程中，主体在二级胺盐和三唑站 点间往复运动, 并伴随着主体和客体之间电子转移作用 的发生.

曲大辉等 ${ }^{[17]}$ 开发了一种电化学响应的[1]轮烷分子 机器, 除了酸碱驱动外, 它还可以在电化学可逆态和电 化学不可逆态之间实现分子开关 (Eq. 10). 当主体 DB24C8 停留在二级胺盐单元上时, 采用循环伏安法可 


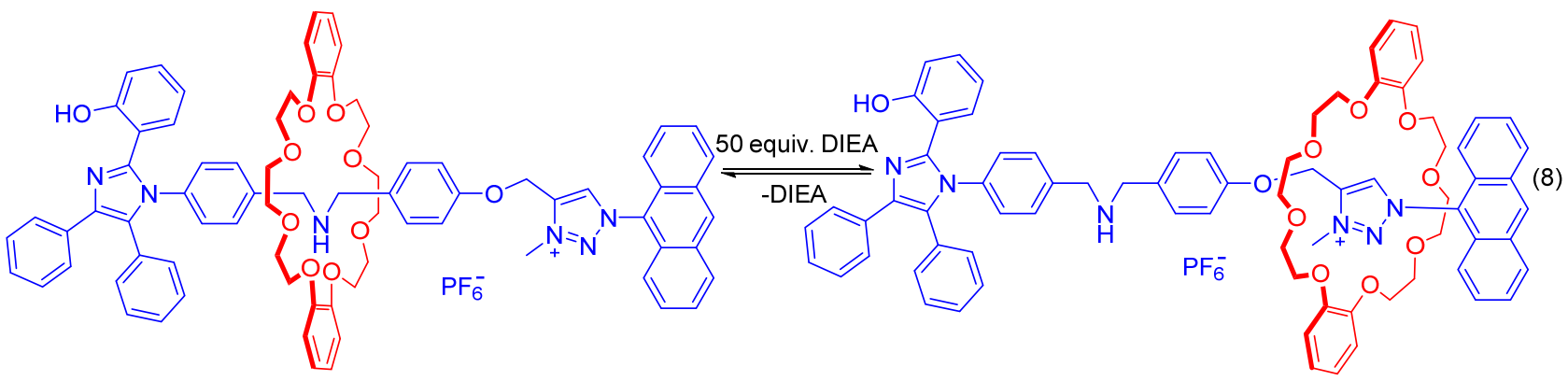

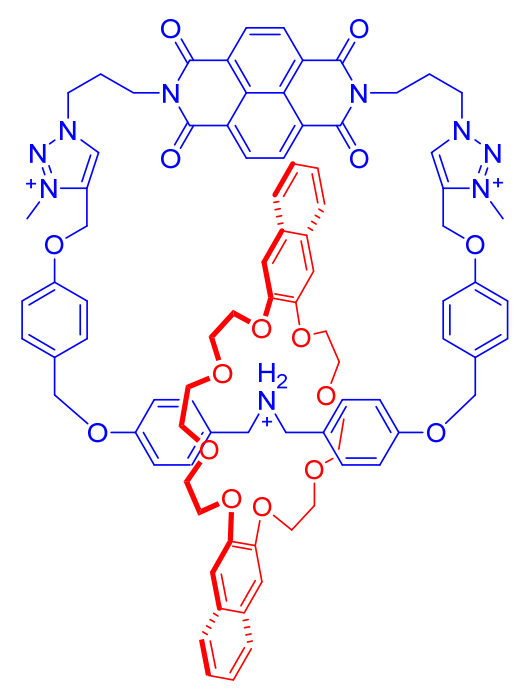

以得到二茂铁单元的氧化-还原曲线; 去质子化后, 大 环移至三唑单元, 氨基外露, 此时不能获得相应的氧化 还原曲线. 这种电化学可逆态和电化学不可逆态之间的 分子开关有望应用于微型材料开关设计.

田禾等 ${ }^{[18]}$ 在大环上引入二茂铁, 采用两种不同的 荧光基团进行封端得到酸碱可控的分子机器和分子开 关(Eq. 11). 随着酸/碱的加入, 主体在客体轴上进行穿 梭运动, 电子给体和不同封端基团之间产生电子转移, 相应的光学信号增强或减弱, 苂光也随之改变. 这种基

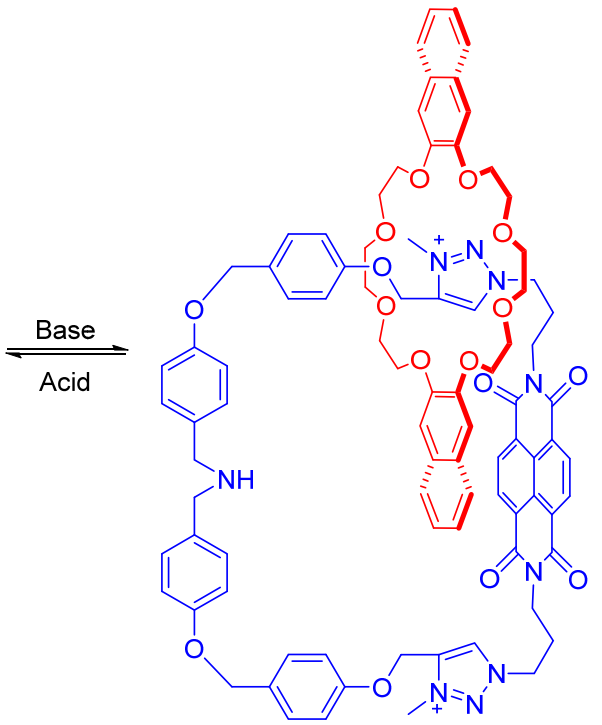

(9)

于酸碱刺激的荧光分子机器有望用于构建光学元件的 分子开关中.

利用空间互补性，刘育小组 ${ }^{[19]}$ 构建了插头-电板型 分子开关(Eq. 12). 这种分子开关能够利用酸碱进行驱 动, 与电器插头的插入-移出非常类似. 由于主体上的 荎基与客体结合后, 具有自敏化光氧化作用, 酸碱的加 入激发了光诱导的电子转移过程, 具有抑制/解除作用, 从而有望成为新型的逻辑门开关.

他们 ${ }^{[20]}$ 还开发了利用酸碱调节分子内部电子转移

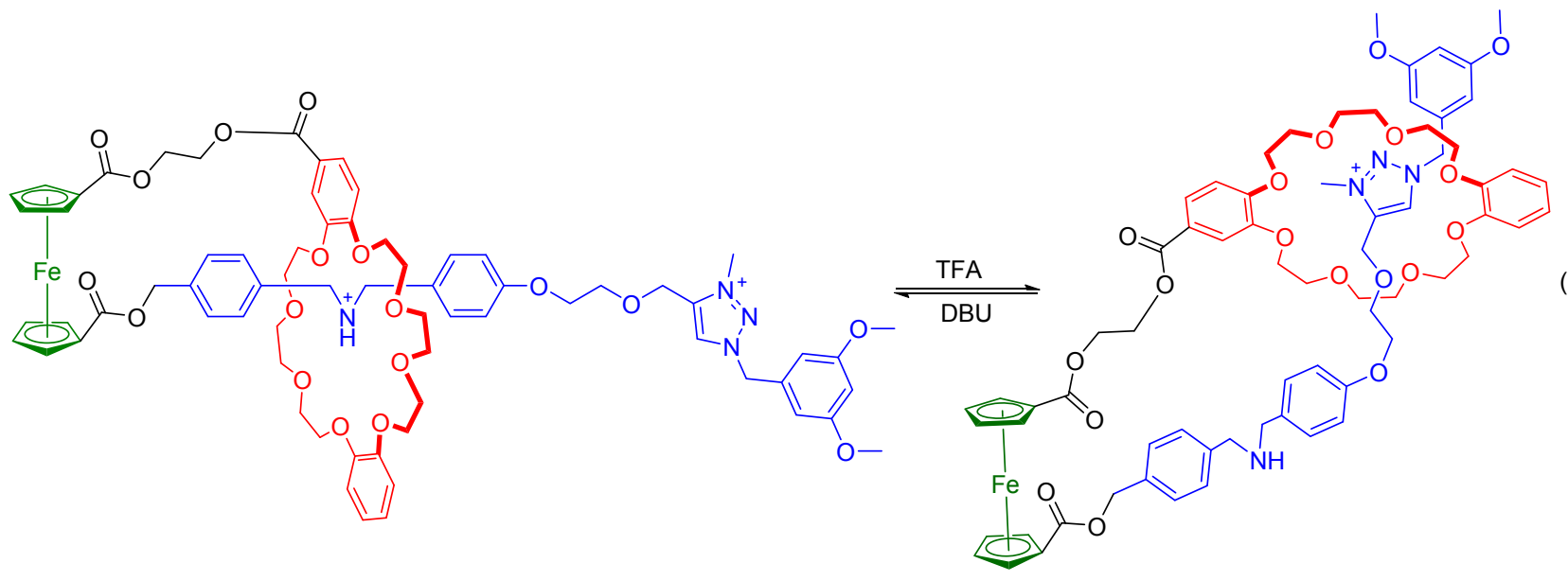



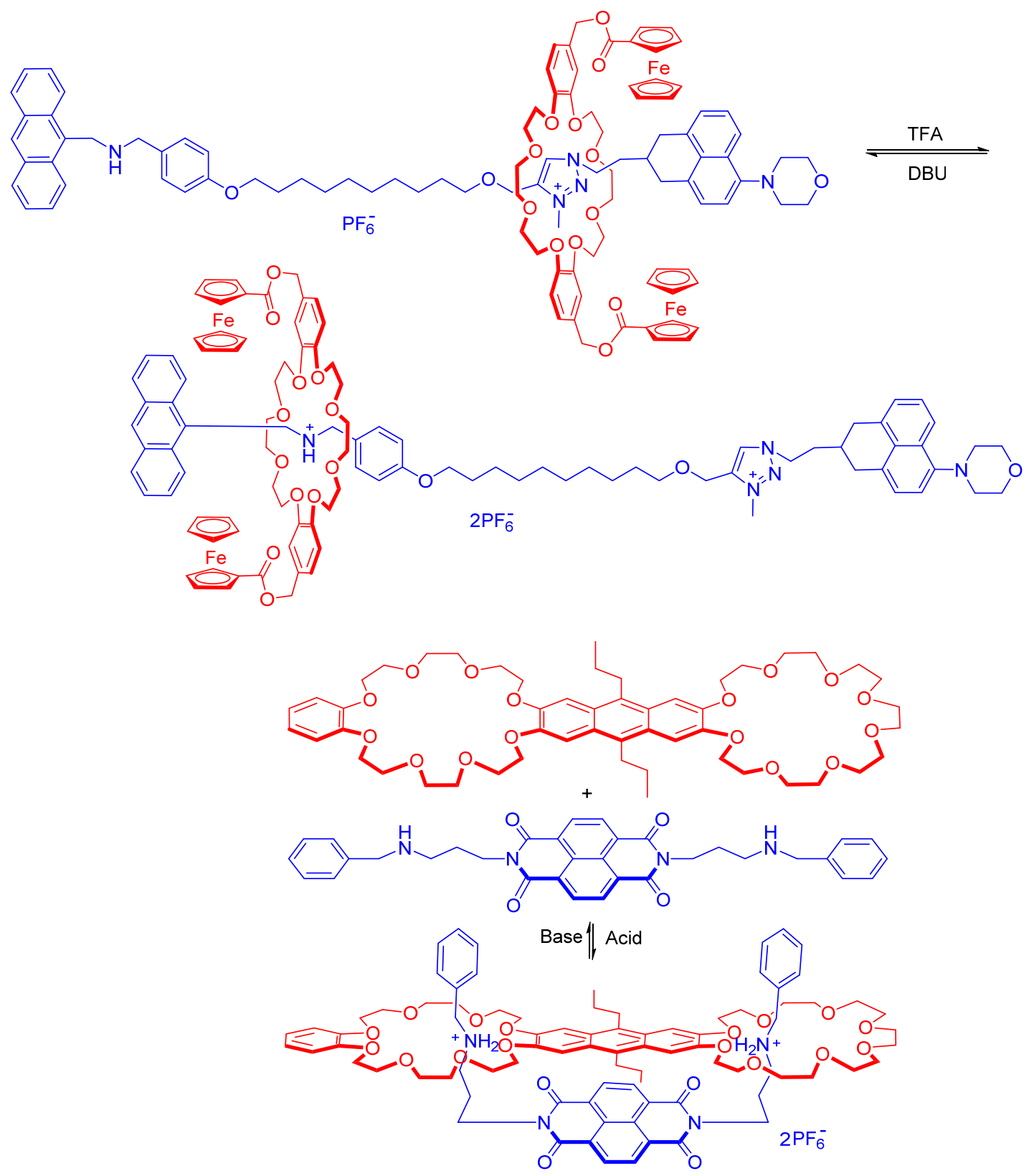

的双稳态分子机器(Eq. 13). 在酸/碱刺激下, 客体上荎 二酰亚胺单元和主体萠单元之间的电子转移过程发生 相应的变化: 去质子化后, 主体从二级胺盐移动至三唑 单元, 电子转移强度增强; 质子化后, 主体回到二级胺 盐单元上, 电子转移强度变弱, 从而成为一种利用酸碱 度调节电子受体和给体之间距离来实现光学性质变化 的分子机器。

李世军小组 ${ }^{[21]}$ 合成了一种新型的吡啶基冠醚, 双 吡啶 30-冠-10 (DP30C10), 并开发了 DP30C10 和 4,4'联吡啶盐、DAP 盐的识别, 利用 DP30C10 中吡啶基的 碱性和 DAP 与有机胺的强络合作用, 实现了基于该识 别的酸/碱、碱/酸双向可调控分子开关. 这种酸/碱调控 分子开关是通过改变主体结构来驱动的(Scheme 4).

\section{2 基于其它大环主体的酸碱调控分子机器与分} 子开关

Stoddart 小组 ${ }^{[22]}$ 制备了以四价阳离子环番为主体的 电化学和酸碱调控双驱动的二站点分子机器(Scheme 5). 联苯胺和联苯二醚分别为该分子机器的识别单元. 在氝代乙腈中, 四价阳离子环番最初优先选择联苯胺上 的识别位点, TFA/吡啶的加入能够驱动四价阳离子环番 在客体中来回往复运动. 此外, 该分子机器也能够通过 电化学驱动.

Leigh 小组 ${ }^{[23]}$ 设计并制备了基于酰胺与二级胺盐的 二站点酸碱调控分子机器(Scheme 6). 由于酰胺-酰胺 的氢键作用, 大环主体稳定在缩肽上; 当二级胺被质子 

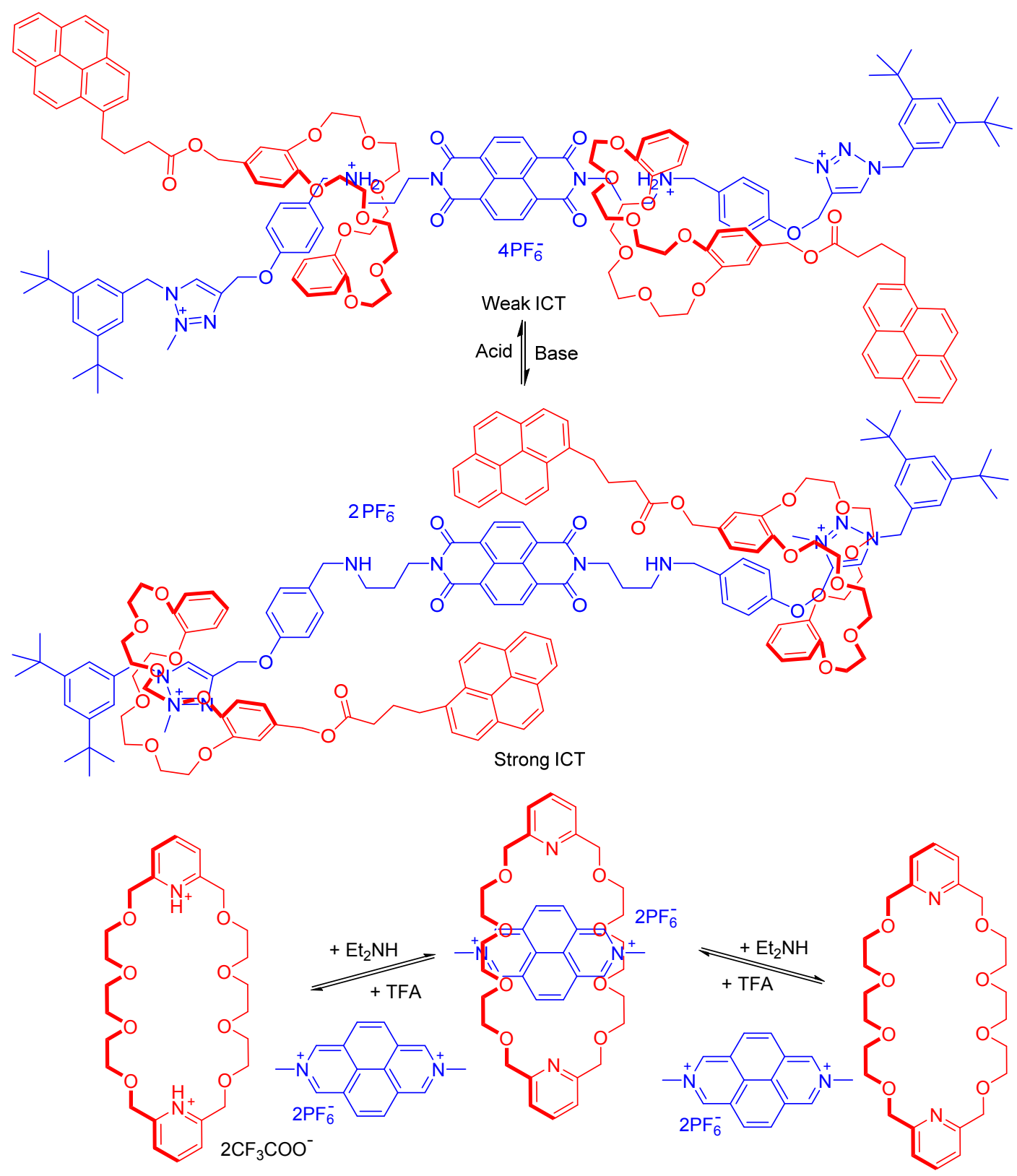

图式 4 基于 $\mathrm{DP} 30 \mathrm{C} 10$ 的酸/碱、碱/酸双向调控运动

Scheme 4 The reversible threading/dethreading processes based on DP30C10

化后, 由于聚醚和二级胺盐阳离子的作用更强, 因此主 体自发向二级胺盐的识别位点上运动. 碱的加入, 使二 级胺盐去质子化, 导致识别位点被移除, 主体逆向运动 回到初始状态. 如此往复, 实现了分子机器的酸碱调控.

邱胜贤等 ${ }^{[24]}$ 研究了酸/碱和金属离子双驱动的分子 机器(Scheme 7). 在加入 $\mathrm{NH}_{4} \mathrm{PF}_{6}$ 时, 主体醚键上的氧和 铵离子形成强的作用力, 将空腔内的 DMDAP 挤出, 实 现主客体分离; 往溶液中加入二胺, 能够竞争络合空腔 内的铵离子, 这样主体与 DMDAP 重新相互识别, 回到 基态, 这是离子调控的驱动机理. 酸碱调控的机理是: 与主体相比, 二级胺更具富电子, 更容易与 DMDAP 络 合, 使得主客体解离; 加酸后, 二级胺被质子化, 主客

\section{体又重新络合.}

李玉良和朱道本等 ${ }^{[25]}$ 制备了一个酸/碱和金属离子 双驱动的苂光分子机器( Scheme 8). 由于 $\mathrm{CH}_{2} \mathrm{NH}_{2}{ }^{+} \mathrm{CH}_{2}$ 和大环聚醚单元之间的 $\left[\mathrm{N}^{+} \cdots \mathrm{H} \cdots \mathrm{O}\right]$ 氢键作用以及主体 和芳基封端单元之间的 $\pi-\pi$ 堆积作用, 主体在开始时处 于二级胺盐的识别位点上; 当酸化后, 主体向酰胺的识 别位点上移动. 此外, 通过主客体中 $\mathrm{O} 、 \mathrm{~N}$ 原子和 $\mathrm{Li}^{+}$、 $\mathrm{Zn}^{+}$离子的可逆络合也能够实现分子梭运动. 在分子梭 运动同时, 封端单元中葱官能团的苂光光谱发生了或强 或弱或淬灭的相应变化, 这使其成为一种多稳态多模式 驱动的荧光响应分子机器. 


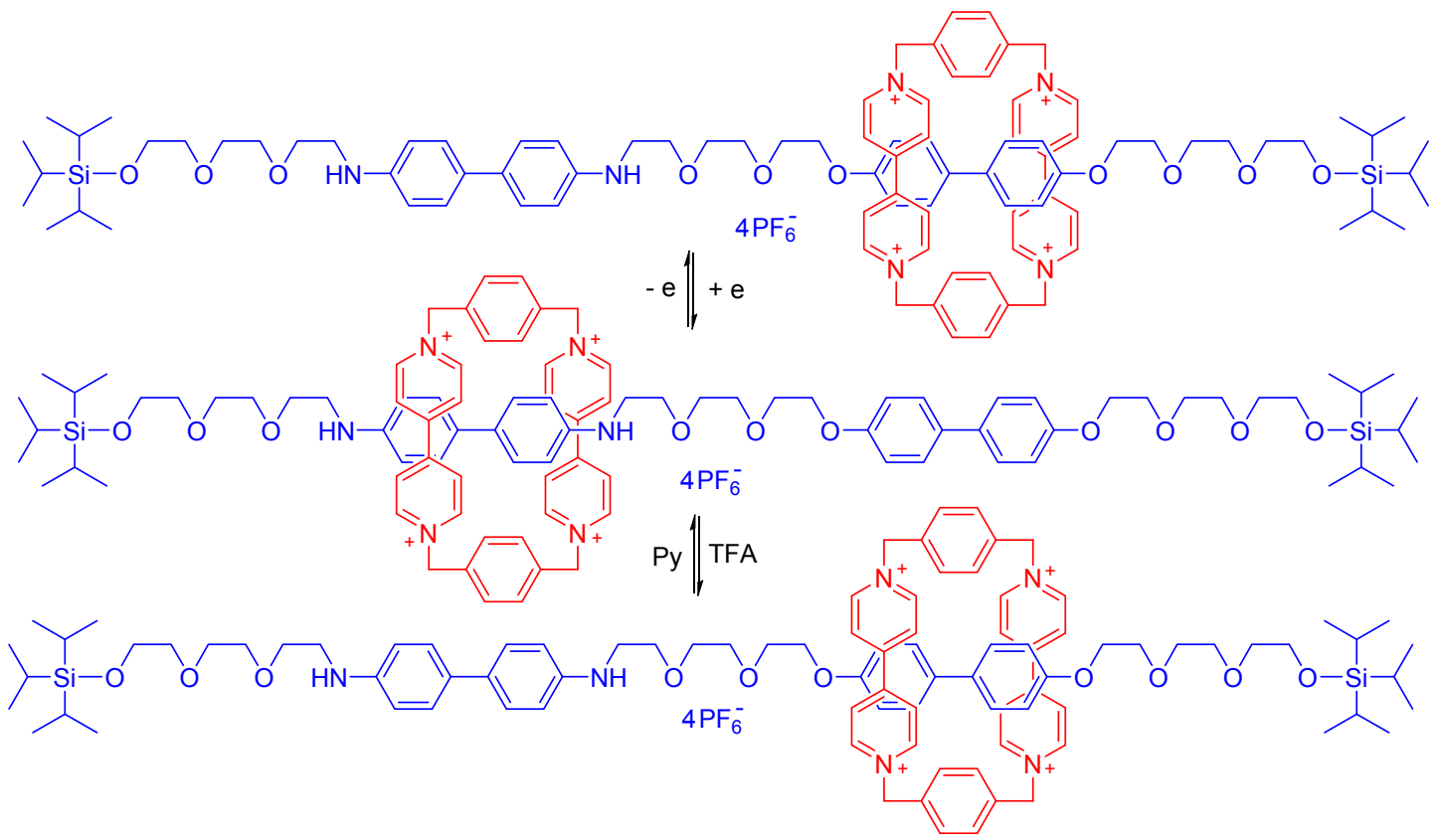

图式 5 酸碱和电化学双驱动的分子机器

Scheme 5 A chemically and electrochemically switchable molecular machine
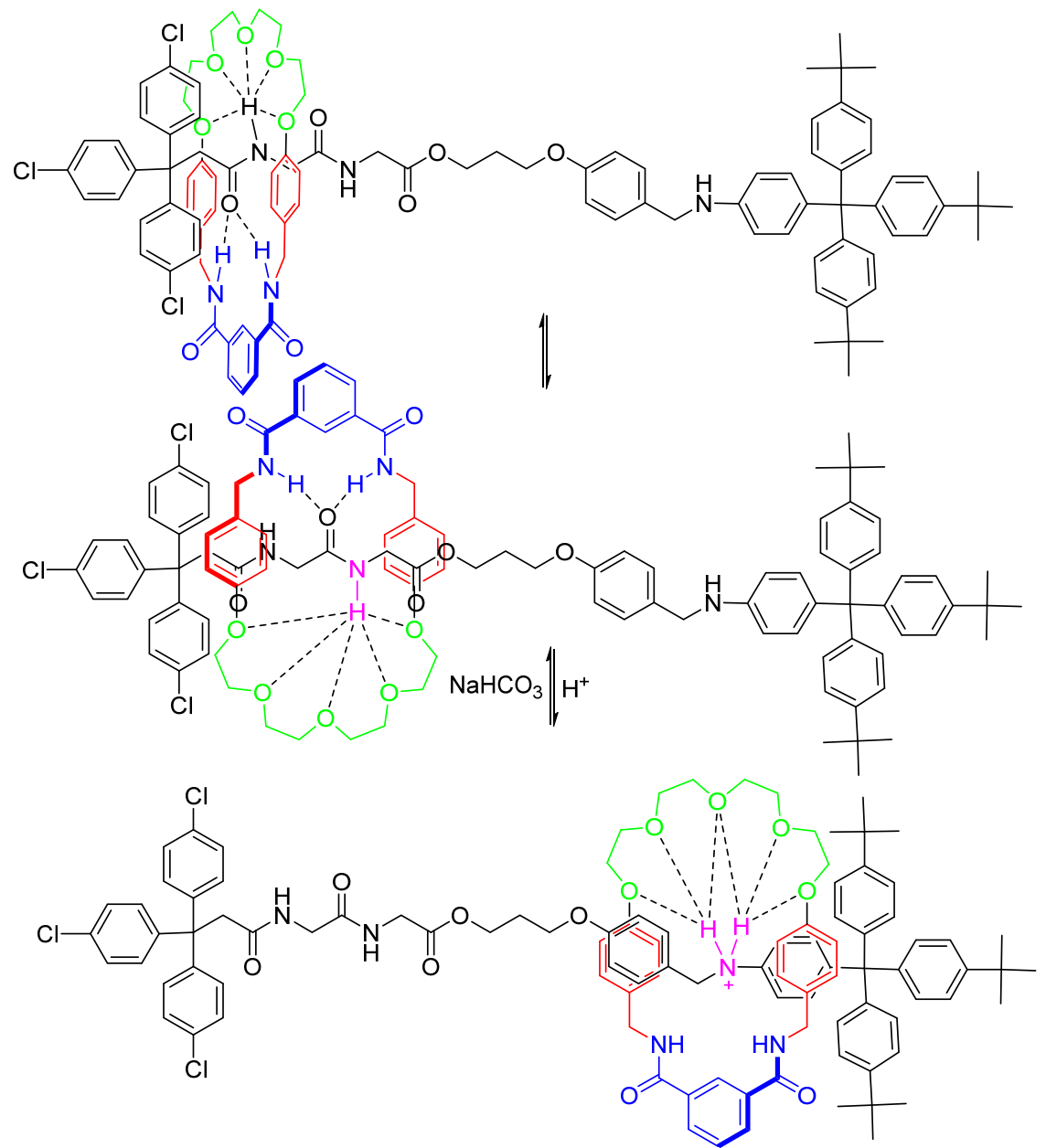

图式 6 基于酰胺与二级胺盐的酸碱调控分子机器

Scheme 6 Switchable dual binding mode molecular machine 

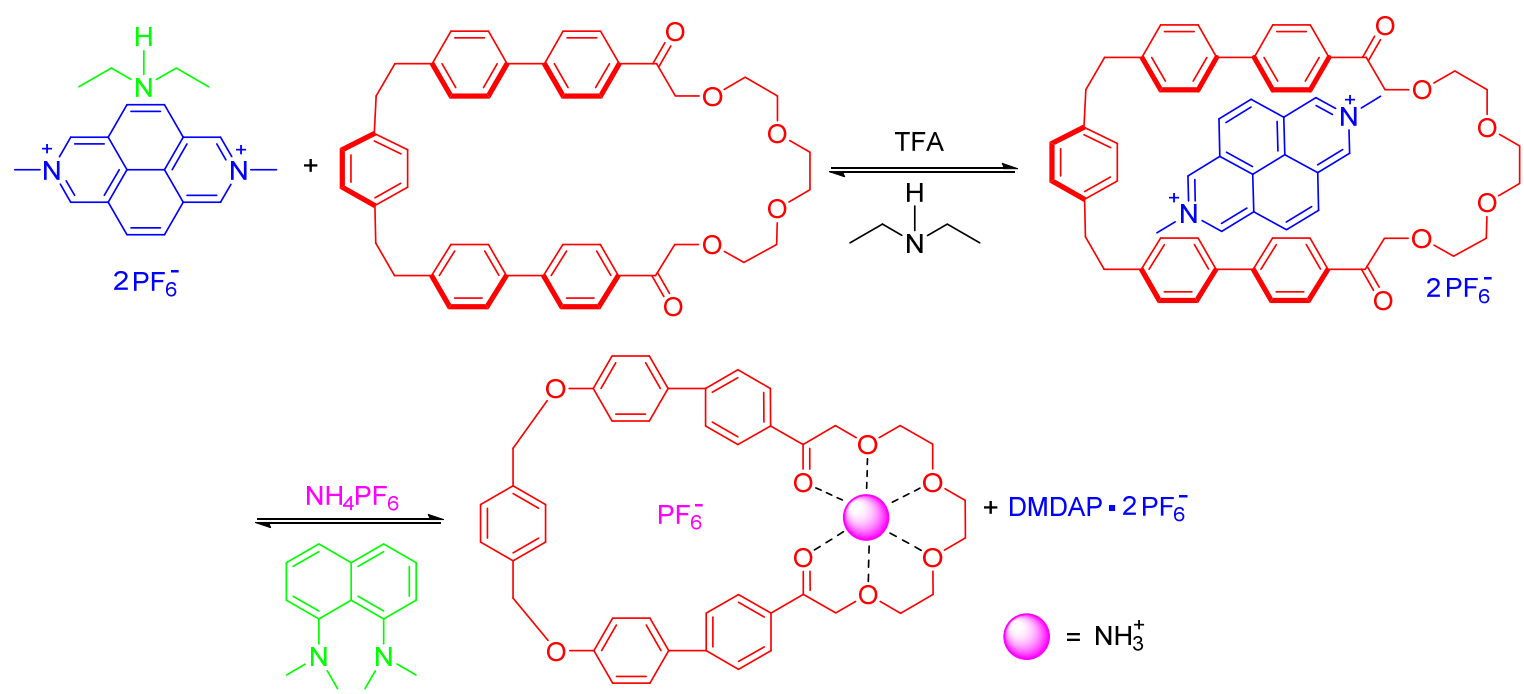

图式 7 酸/碱和铵盐双驱动的可控识别

Scheme 7 Dual-action acid/base- and base/acid-controllable molecular switch

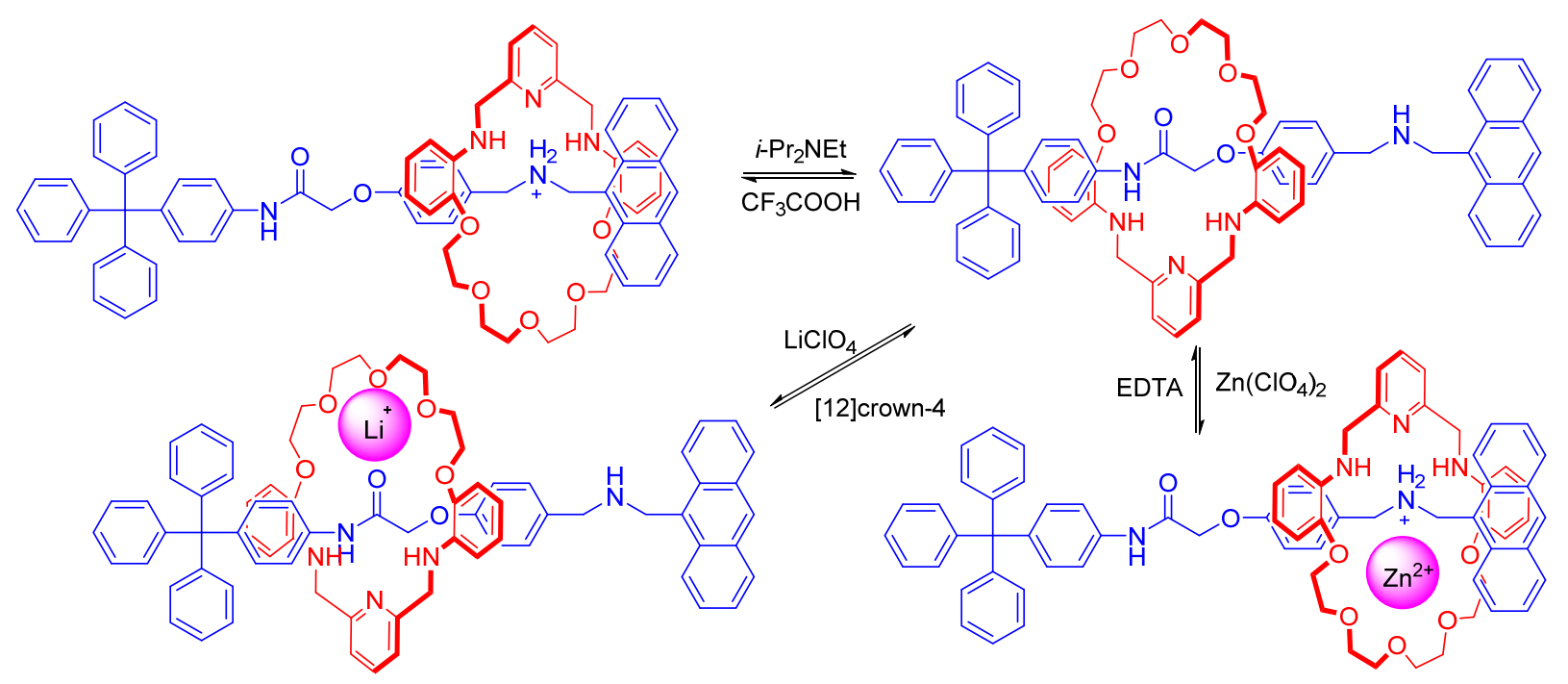

图式 8 酸/碱和金属盐双驱动的分子机器

Scheme 8 A molecular machine for driving a multilevel fluorescence switch

李玉良等 ${ }^{[26,27]}$ 利用 $\mathrm{Cu}$ 催化的 1,3-偶极环加成反应 开发了一系列酸碱可控的分子机器. Scheme 9 中的 [2]轮 烷质子化后, 主体因与二级胺盐具有更高的络合常数停 留在该部位; 加入 $\mathrm{Et}_{3} \mathrm{~N}$ 将二级胺盐去质子后, 主体往三 唑方向运动; 而加入氯离子后, 大环酰胺和三唑协同与 氯离子进行识别, 从而导致大环发生旋转运动.

在此基础上, 他们 ${ }^{[27]}$ 引入具有苂光性质的封端基 团来获得此类分子机器的刺激/响应功能(Scheme 10). 基于不同识别位点的络合能力差别, 大环主体优先被固 定在二级胺盐位点上, 在荧光信号上表现为中等强度; 加入 $\mathrm{Et}_{3} \mathrm{~N}$ 去质子化后, 主体移动到三唑站点上, 伴随着 荧光信号的明显增强. 当加入更强的碱 Schwesinger's P1 base 后, 封端基团咪唑衍生物上的酚羟基变成氧负 离子, 大环酰胺与它形成氢键作用, 因而滑移至封端基
团附近，而苂光信号表现为最弱. 酸化溶液后，主体回 到初始状态, 苂光信号增加至中等强度. 这种利用酸碱 刺激来实现分子运动并伴随着荧光应答的方式有望于 应用到多稳态苂光探测体系中.

三桥大环主体与 paraquat 盐的强络合能力被黄飞鹤 和 Gibson 等 ${ }^{[28]}$ 应用到酸碱调控的分子机器中(Scheme 11). 吡啶衍生化的三桥主体与 paraquat 盐由于在空间和 能量上更加匹配具有很高的络合常数, 并且还利用吡啶 基的性质实现了基于 cryptand/paraquat 识别的酸碱驱动 分子梭运动.

刘育小组 ${ }^{[29]}$ 研究了 [8]葫芦腿和双 4,4'-联吡啶衍生 物的酸碱调控分子自组装过程(Eq. 14). [8]葫芦脲和客 体双 4,4'-联吡啶衍生物自组装形成稳定的包含物(夹心 结构); 当酸化联吡啶后, [8]葫芦腿和客体形成互穿的准 


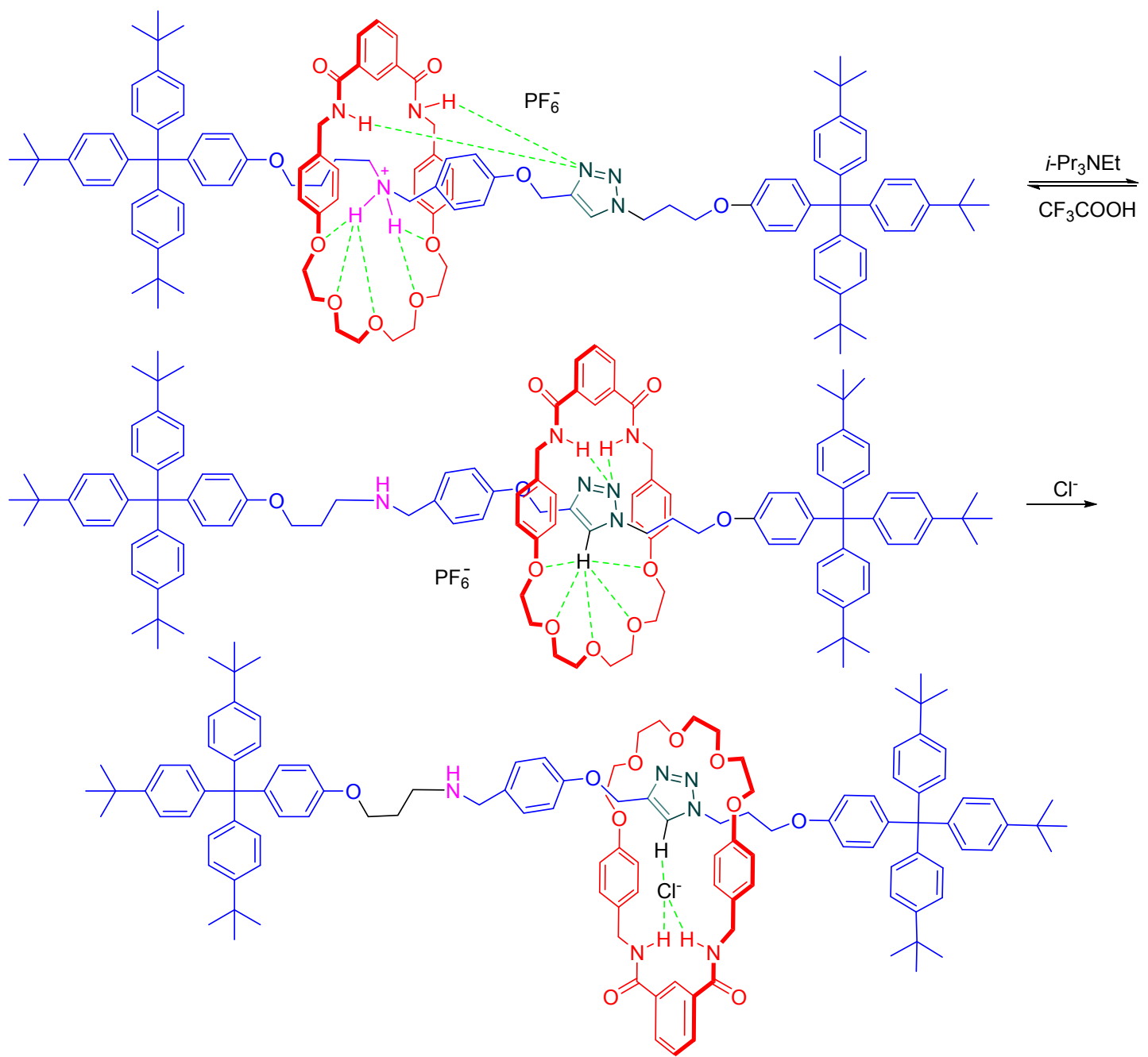

图式 9 含三唑单元轮烷的穿梭运动和结构变化示意图

Scheme 9 Schematic representation of shuttling and conformation variation of the rotaxane-containing triazole station

轮烷结构, 而去质子化后又变回到夹心态. 这种分子机 器能在中性/酸性条件下可逆进行, 表现出良好的电化 学可控性和光谱性.

陈传峰小组 ${ }^{[30]}$ 通过 click 反应制备了基于三叠烯四 桥大环主体和含有二级胺盐、三唑盐双识别位点的[3] 轮烷分子机器(图 1). 这种酸碱调控的 [3]轮烷分子机器 的成功开发对多组分同时运动的复杂分子机器构建具 有重要意义.

此外, 他们 ${ }^{[31]}$ 研究了一种基于三碟烯的新型大环 主体和系列百草枯客体络合形成准轮烷结构(图 2). 缺 电子的 4,4'-联吡啶盐和富电子的 1,8 -二氮荎单元之间的 作用，使得主客体络合; 主体上的氮原子被质子化后， 主客体之间产生电荷排斥而发生解离. 除了酸碱可 调控外, 金属离子 $\mathrm{Hg}^{2+}$ 也能实现调控. 1,8-二氮荎单 元属于双齿配体, 能够对 $\mathrm{Hg}^{2+}$ 离子进行配位. 在上述 准轮烷中, 加入 $\mathrm{Hg}^{2+}$ 离子, 大环被络合, 主客体分离; 当加入 $\mathrm{KI}$ 清除 $\mathrm{Hg}^{2+}$ 后, 主客体重新络合. 这种运动
过程在苂光谱图中也得到了验证, $\mathrm{Hg}^{2+}$ 离子的加入/ 清除带来了荧光的消失/恢复, 而成为一种苂光开 关 $^{[30]}$.

\section{3 酸碱调控分子机器与分子开关的应用}

为了实现分子机器在纳米器件及药物传递等领域 的应用, 近年来, Stoddart 小组和 Zink 小组 ${ }^{[32 \sim 34]}$ 协作致 力于开发支载于纳米颗粒的酸碱调控分子机器, 并将其 应用到药物可控释放. 通过控制支载于介孔二氧化硅等 材料表面的分子机器或分子开关, 实现吸附在纳米粒子 中药物的可控释放, 目前他们已在这方面取得了瞩目的 成果 ${ }^{[32 \sim 39]}$.

例如，他们 ${ }^{[33]}$ 制备了基于 $\mathrm{CB}[6] /$ 二级胺盐准轮烷的 酸碱可控机械化纳米粒子系统(MNPs)(图 3). 在中性环 境下, 药物被介孔二氧化硅材料捕获后, 加入 $\mathrm{CB}[6]$, 由于客体末端基团小, 在 4,4'-联吡啶的识别作用下, $\mathrm{CB}[6]$ 穿过客体末端, 往材料表面移动, 封锁了药物的 


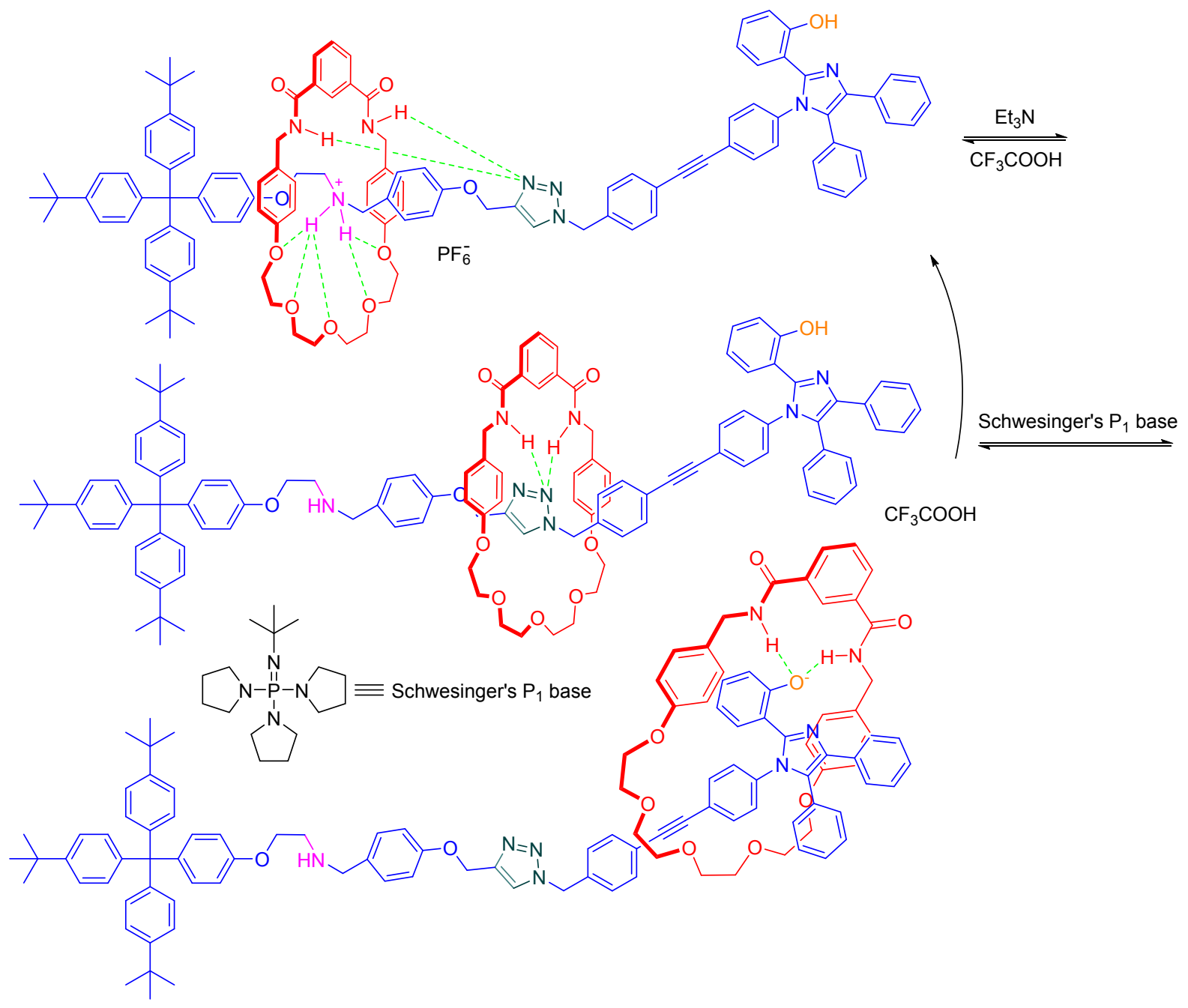

图式 10 [2]轮烷在不同条件刺激下的分子梭运动

Scheme 10 The shuttling movement process of the [2]rotaxane under different stimuli

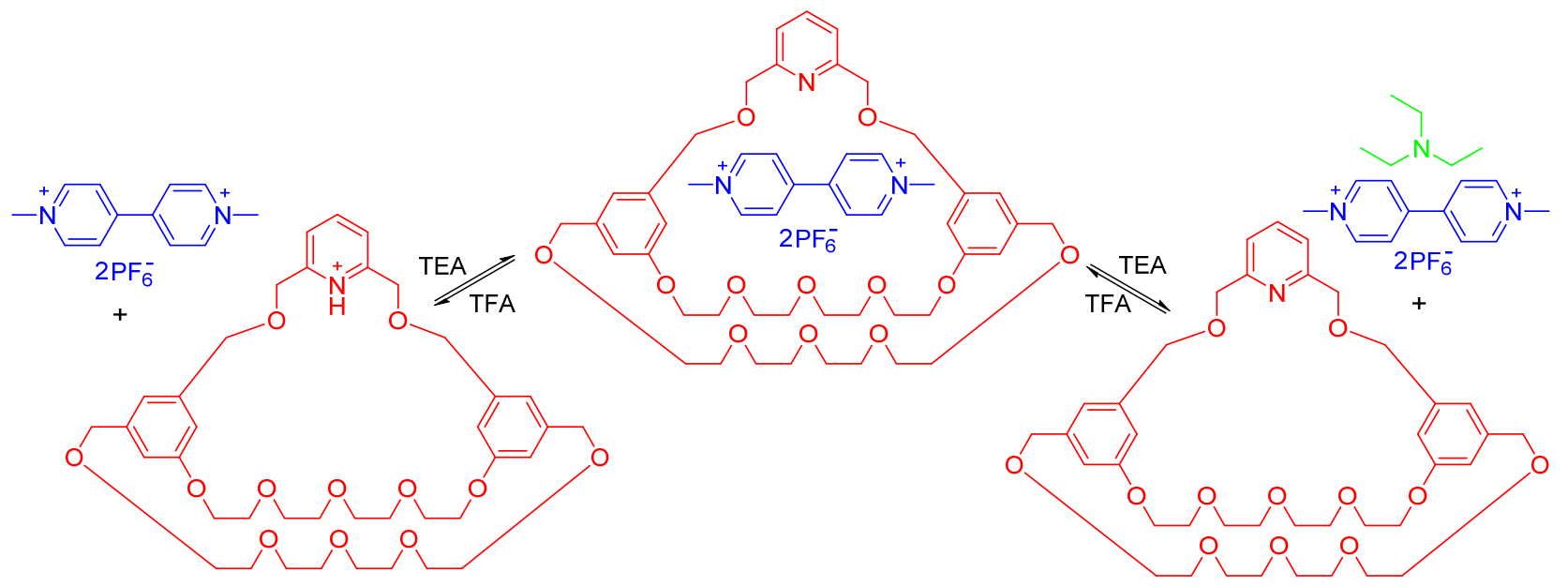

图式 $11 \mathrm{pH}$-调控的基于三桥大环主体/百草枯[2]准轮烷的组装与解离

Scheme 11 pH-controlled assembly and disassembly of a cryptand/paraquat[2]pseudorotaxane

释放通道, 进而将负载的药物牢牢地堵在材料内部. 当 $\mathrm{pH}$ 低于 6 时, 二级胺被质子化后, $\mathrm{CB}[6]$ 往成二级胺盐 识别位点移动, 通道被打开, 药物获得释放, 从而可实
现酸碱调控的靶向治疗. 这个体系的优点在于大环主体 $\mathrm{CB}[6]$ 和线性客体上的单元及末端基团具有高度的水溶 性, 是分子机器在酸性水溶液环境中成功运转的至关重 

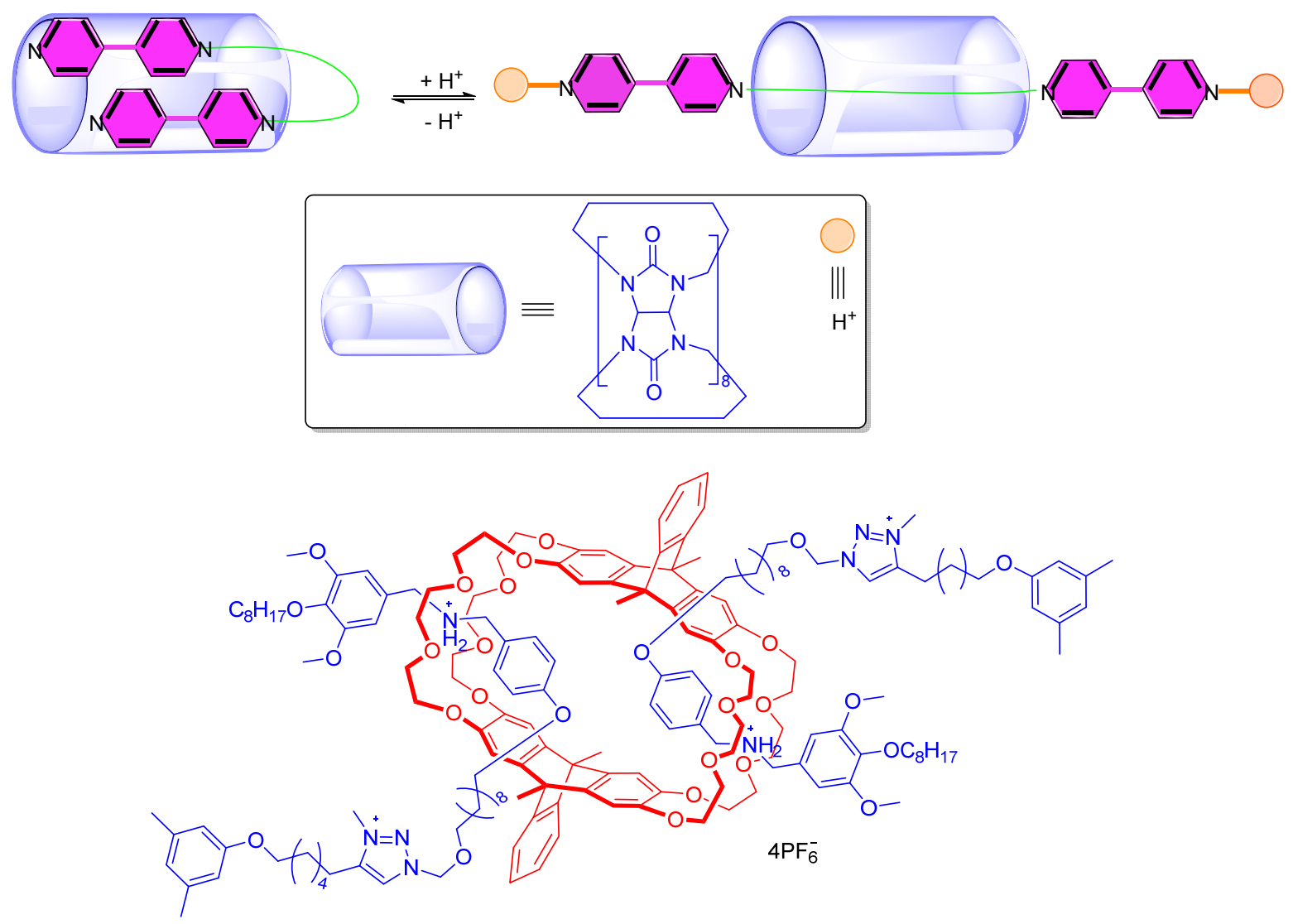

图 1 基于二苄胺盐和三坐位点的[3]轮烷的分子机器

Figure 1 A [3] rotaxane molecular machine based on a dibenzylammonium ion and a triazolium station

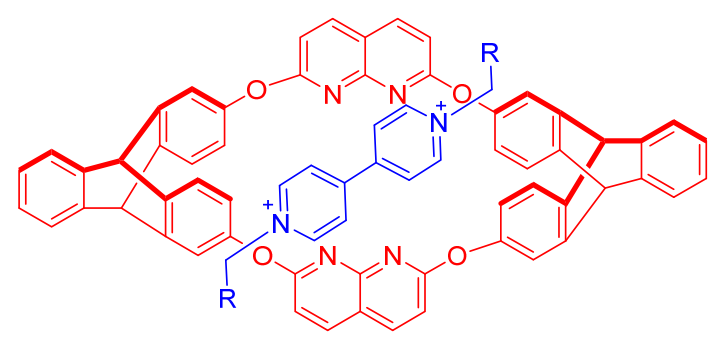

$\mathrm{R}=\mathrm{H}, \mathrm{CH}=\mathrm{CH}_{2}, \mathrm{CH}_{2} \mathrm{OH},\left(\mathrm{CH}_{2}\right)_{4} \mathrm{CH}_{2} \mathrm{OH}, \mathrm{CH}_{2} \mathrm{OCH}_{3}$, $\mathrm{CH}_{2} \mathrm{OCH}_{2} \mathrm{CH}=\mathrm{CH}_{2}, \mathrm{CH}_{2} \mathrm{OCH}_{2} \mathrm{CCH}$

图 $2 \mathrm{pH}$-调控的[2]准轮烷的组装与解离

Figure 2 pH-controlled assembly and disassembly of a [2]pseudorotaxane

要的一例. 它的出现为改进生物兼容性的新型药物载体 奠定了基础

另外, 他们基于 $\mathrm{CB}[6] /$ 三胺盐准轮烷的双稳态主客 体识别制备了酸/碱双向调控的药物释放体系 ${ }^{[34]}$. 先对 二氧化硅纳米粒子表面进行修饰, 将三胺盐杆状分子支 载在纳米粒子表面. 通过将三胺盐分子链接的纳米颗粒 浸泡在碘化丙啶(PI)的溶液中使得染料分子扩散到空的 纳米小孔中, 从而完成染料的载入. 然后, 通过向染料 载入的纳米颗粒混合物中加入 $\mathrm{CB}[6]$, 使得 $\mathrm{CB}[6]$ 环与 三胺盐杆状分子之间发生络合, 完成准轮烷和机械化纳
米颗粒体系的制备, 开发了基于的机械化纳米颗粒 ${ }^{[34]}$ (图 4). 这里的机械化纳米颗粒的运转有三个重要的设 计特点: (1)苯胺的氮原子与其他两个氮原子存在着碱度 的差异，(2)客体分子被三个胺基分段的长度不同; (3)不 同的官能团 $\mathrm{R}$ 使得末端胺基具有不同的酸碱性. 与烷基 的氮原子相比, 苯胺的氮原子的碱性要弱近 106 倍, 导 致其在中性 $\mathrm{pH}$ 值环境下不能被质子化. 在中性 $\mathrm{pH}$ 值 下, $\mathrm{CB}[6]$ 环环绕在四亚甲基二胺的位置周围, 大环两端 都能够参与离子一偶极结合的相互作用, 从而阻塞小孔 的孔口并且封住客体分子，机械化纳米粒子处在其关闭 的形态. 相比 $\mathrm{NH}_{3}^{+}\left(\mathrm{CH}_{2}\right)_{4} \mathrm{NH}_{3}^{+}$对大环的识别, $\mathrm{NH}_{3}^{+}\left(\mathrm{CH}_{2}\right)_{6} \mathrm{NH}_{3}^{+}$具有更稳定的络合能力. 不仅如此, 六碳的间隔与 $\mathrm{CB}[6]$ 的内在尺寸是一个较好的匹配, 因 而, 一旦 $\mathrm{pH}$ 值降低, 则苯胺的氮原子被质子化, $\mathrm{CB}[6]$ 环可以穿梭于末端四亚甲基二胺的识别单元, 纳米孔口 被疏通，封锁在内部的物质得以释放出来. 此外，通过 对末端取代的 $\mathrm{R}$ 基团进行相平行的化学修饰，来调整苯 胺氮原子的酸离解常数 $\left(\mathrm{p} K_{\mathrm{a}}\right)$, 从而使得用来打开机械 化纳米颗粒所需的 $\mathrm{pH}$ 值可以被调节. 他们列举了 $\mathrm{H}(\mathrm{MNP}-1)$ 和 $\mathrm{OCH}_{3}(\mathrm{MNP}-2)$ 两种不同取代的机械化纳米 颗粒系统的释放性能, 发现其相应的 $\mathrm{pH}$ 值有一定的差 


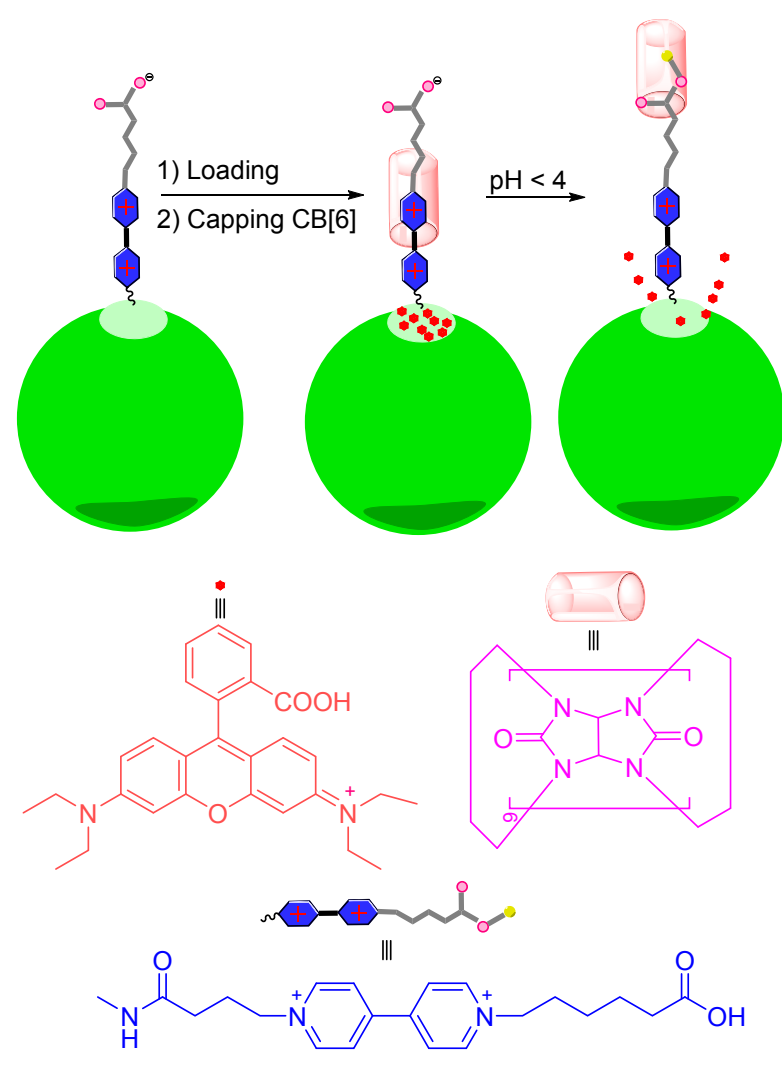

图 $3 \mathrm{pH}$ 调控的机械化纳米粒子系统

Figure 3 pH-controlled MNPs

异, 证明了机械化纳米颗粒系统响应的 $\mathrm{pH}$ 值可以通过 对杆状分子进行合理的化学修饰而进行调节. 简而言 之, 通过在不同组织中使用不同的 $\mathrm{pH}$ 值从而增强机械 化纳米颗粒系统的灵活性, 可以实现微调, 使其处于最 佳应答状态, 从而将机械化纳米颗粒运用到治疗不同的 癌症细胞中.

像这样, 分子机器被吸附在介孔二氧化硅等材料 后, 使其从无序化运动变为朝着有序的方向运动, 实现 了通过外界刺激或环境酸碱度的改变使药物从载体上 释放出来, 从而达到靶向治疗的效果. 此外, 在可控超 分子聚合物与分子机器的宏观化材料构筑及应用方面 已取得了卓越的成效 ${ }^{[40 \sim 43]}$. 尽管这些应用尚处于发展 的起步阶段, 但相信经过纳米技术和分子机器技术的进 一步发展与完善, 将最终真正受益于社会.

\section{4 总结和展望}

综上所述, 各种溶液酸碱度驱动的分子机器已被成 果地制备, 而且所制备的分子机器也越来越呈现出复杂 性、多样性、可控性. 以 Stoddart 等为代表的科学家们 正试图对分子机器进行有序排列, 以 “从底部构筑的方 法” 制造功能效用稳定、成本合适的纳米器件, 用于信 息存储、电子器件、药物传递等诸多方面. 分子机器对

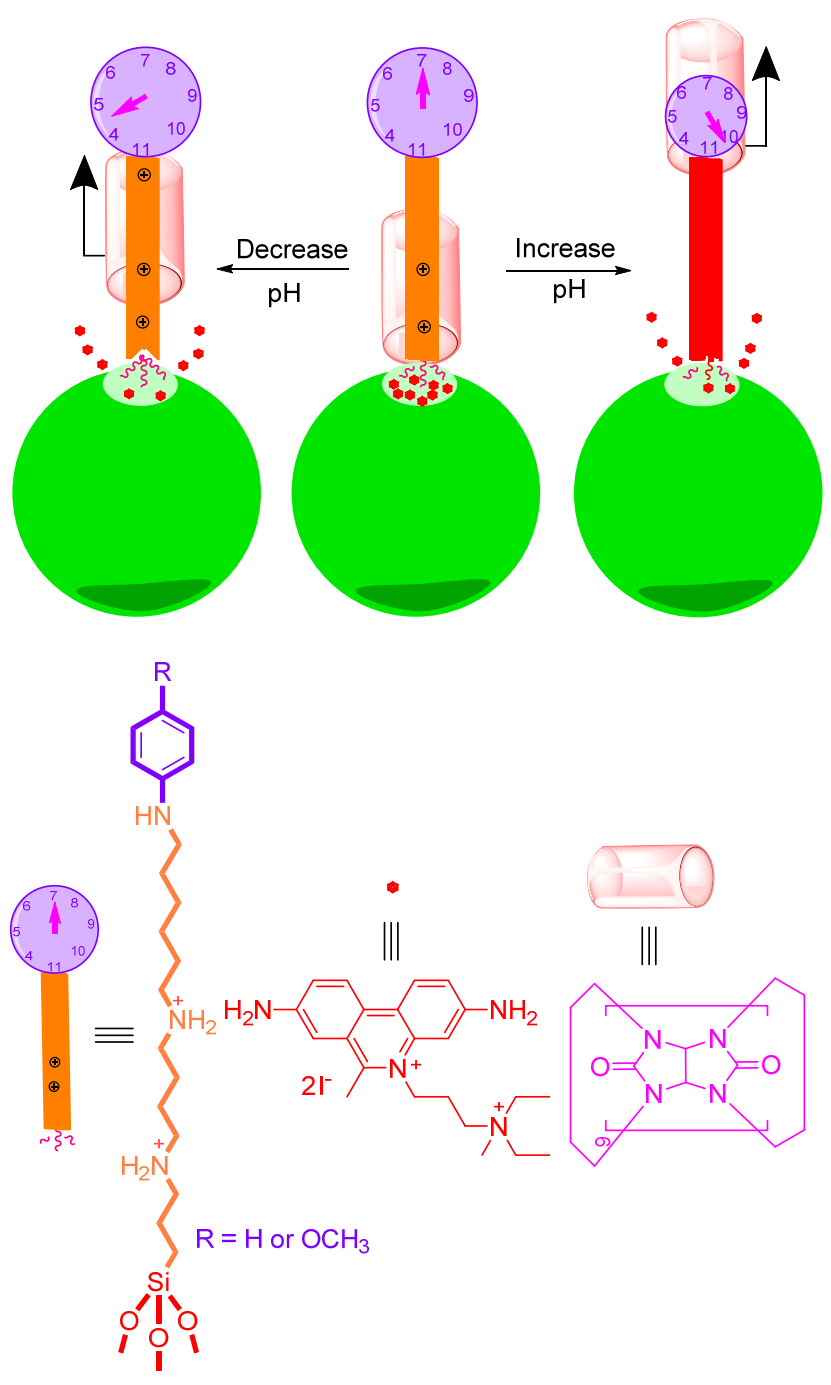

图 $4 \mathrm{pH}$ 值时钟控制的机械化纳米粒子

Figure 4 pH clock-operated MNPs

于研究与模拟生物领域的超分子过程(如光合作用、酶 的催化作用等)也有着十分重要的意义.

但是, 在现阶段分子机器研究中仍然存在着一定的 缺陷. 目前, 分子机器的驱动、表征, 大多都是在溶液中 进行的. 由于无法很好地控制溶液, 分子机器存在杂乱 无章的、不易控制的布朗运动，因而根本无法规整有序 地控制分子机器运动. 因此, 一些科学家提出, 下一代 分子机器必须能使分子在固态表面进行有序排列. 只有 将分子机器合成与纳米科学中分子的有序排列结合起 来, 才能真正地实现用分子来构建纳米器件. 在近年的 研究中, 化学家已经开始尝试将分子机器制成 LB 薄膜, 或者在固体表面将分子机器有序排列. 经过不解努力, 相信在不久的将来分子机器可望能够真正在实际应用 中体现其特有的价值. 


\section{References}

[1] (a) Balzani, V.; Credi, A.; Raymo, F. M.; Stoddart, J. F. Angew. Chem. Int. Ed. 2000, 39, 3348.

(b) Kinbara, K.; Aida, T. Chem. Rev. 2005, 105, 1377.

(c) Ballardini, R.; Balzani, V.; Credi, A.; Gandolfi, M. T.; Venturi, M. Acc. Chem. Res. 2001, 34, 445 .

(d) Champin, B.; Mobian, P.; Sauvage, J. P. Chem. Soc. Rev. 2007, 36,358 .

(e) Ma, X.; Wang, Q. C.; Tian, H. Process Chem. 2009, 21, 106 (in Chinese).

(马骧, 王巧纯, 田禾, 化学进展, 2009, 21, 106.)

(f) Kay, E. R.; Leigh, D. A.; Zerbetto, F. Angew. Chem., Int. Ed. 2007, 46, 72 .

(g) Li, H.; Xu, X. C.; Chen, J. W.; Yang, C. L.; Qing, J. G. Chin. J. Org. Chem. 2008, 28, 2057 (in Chinese).

(李昊, 许犧晨, 陈嘉伟, 杨楚罗, 秦金贵, 有机化学, 2008, 28, 2057.)

(h) Li, S.; Huang, J.; Cook, T. R.; Pollock, B. J.; Kim, H.; Chi, K.-W.; Stang, P. J. J. Am. Chem. Soc. 2013, 135, 2084.

(i) Li, S.; Huang, J.; Zhou, F.; Cook, T. R.; Yan, X.; Ye, Y.; Zhu, B.; Zheng, B.; Stang, P. J. J. Am. Chem. Soc. 2014, 136, 5908.

(j) Zhou, D. X.; Sun, T.; Deng, W. Chin. J. Org. Chem. 2012, 32, 239 (in Chinese).

(周冬香, 孙涛, 邓维, 有机化学, 2012, 32, 239.)

(k) Fan, C. H.; Yang, Y. Q.; Zhao, W.; Xiao, Y.; Luo, J.; Liu, X. Y. Acta Chim. Sinica 2013, 71, 934 (in Chinese).

(范存华, 杨逸群, 赵伟, 肖宇, 罗静, 刘晓亚, 化学学报, 2013, 71, 934.)

(1) Han, Y.; Guo, J. B.; Chen, C. F. Chin. J. Chem. 2014, 32, 721.

(m) Yue, S. Y.; Zhou, Y. J.; Yao, Y.; Xue, M. Acta Chim. Sinica 2014, 72, 1053 (in Chinese)

(岳诗雨, 周玉娟, 姚勇, 薛敏, 化学学报, 2014, 72, 1053.)

[2] (a) Dong, S. Y.; Luo, Y.; Yan, X. Z.; Zheng, B.; Ding, X.; Yu, Y. H.; Ma, Z.; Zhao, Q. L.; Huang, F. H. Angew. Chem., Int. Ed. 2011, 50, 1905.

(b) Zhang, M. M.; Xu, D. H.; Yan, X. Z.; Chen, J. Z.; Dong, S. Y.; Zheng, B.; Huang, F. H. Angew. Chem., Int. Ed. 2012, 51, 7011.

(c) Yan, X. Z.; Xu, D. H.; Chi, X. D.; Chen, J. Z.; Dong, S. Y.; Ding, X.; Yu, Y. H.; Huang, F. H. Adv. Mater. 2012, 24, 362.

(d) Yang, W. L.; Li, Y. J.; Zhang, J. H.; Chen, N.; Chen, S. H.; Liu, H. B.; Li, Y. L. Small 2012, 8, 2602.

(e) Yang, W. L.; Li, Y. J.; Liu, H. B.; Chi, L. F.; Li, Y. L. Small 2012, 8, 504 .

(f) Li, Y. J.; Liu, H. B.; Li, Y. L. Curr. Org. Chem. 2011, 15, 97.

[3] (a) Wang, F.; Han, C. Y.; He, C. L.; Zhou, Q. Z.; Zhang, J. Q.; Wang, C.; Li, N.; Huang, F. H. J. Am. Chem. Soc. 2008, 130, 11254. (b) Dong, S. Y.; Zheng, B.; Xu, D. H.; Yan, X. Z.; Zhang, M. M.; Huang, F. H. Adv. Mater. 2012, 24, 3191.

[4] Ashton, P. R.; Ballardini, R.; Balzani, V.; Baxter, I.; Credi, A.; Fyfe, M. C. T.; Gandolfi, M. T.; Gomez-Lopez, M.; Martine-Diaz, M.; Piersanti, A.; Spencer, N.; Stoddart, J. F.; Venturi, M.; White, A. J. P.; Williams, D. J. J. Am. Chem. Soc. 1998, 120, 1932.

[5] Ashton, P. R.; Baldoni, V.; Balzani, V.; Credi, A.; Hoffmann, H. D. A.; Martinez, D. M.; Raymo, F. M.; Stoddart, J. F.; Venturi, M. Chem. Eur. J. 2001, 16, 3482.

[6] (a) Badjic, J. D.; Balzani, V.; Credi, A.; Silvi, S.; Stoddart, J. F. Science 2004, 303, 1845.

(b) Badjic, J. D.; Ronconi, C. M.; Stoddart, J. F.; Balzani, V.; Silvi, S.; Credi, A. J. Am. Chem. Soc. 2006, 128, 1489.

[7] (a) Wu, J. S.; Leung, K. C. F.; Benítez, D.; Han, J. Y.; Cantrill, S. J.; Fang, L.; Stoddart, J. F. Angew. Chem., Int. Ed. 2008, 47, 7470. (b) Fang, L.; Hmadeh, M.; Wu, J.; Olson, M. A.; Spruell, J. M.; Trabolsi, A.; Yang, Y. W.; Elhabiri, M.; Albrecht-Gary, A.; Stoddart, J. F. J. Am. Chem. Soc. 2009, 131, 7126.

[8] Du, G.; Moulin, E.; Jouault, N.; Buhler, E.; Giuseppone, N. Angew. Chem., Int. Ed. 2012, 51, 12504.

[9] Leigh, D. A.; Thomson, A. R. Tetrahedron. 2008, 64, 8411.

[10] Coutrot, F.; Eric, B. Chem. Eur. J. 2008, 14, 4784

[11] Clavel, C.; Romuald, C.; Brabet, E.; Coutrot, F. Chem. Eur. J. 2013, 19, 2982.

[12] (a) Credit, A.; Balzani, V.; Langford, S. J.; Stoddart, J. F. J. Am. Chem. Soc. 1997, 119, 2679.

(b) Asakawa, M.; Iqbal, S.; Stoddart, J. F.; Tinker, N. D. Angew. Chem., Int. Ed. 1996, 35, 976.

(c) Ballardini, R.; Balzani, V.; Credi, A.; Gandolfi, M. T.; Langford, S. J.; Menzer, S.; Prodi, L.; Stoddart, J. F.; Venturi, M.; Williams, D. J. Angew. Chem., Int. Ed. 1996, 35, 978.

[13] Yan, X. Z.; Wu, X. J.; Wei, P. F.; Zhang, M. M.; Huang, F. H. Chem. Commun. 2012, 48, 8201.

[14] Yan, X. Z.; Zhang, M. M.; Wei, P. F.; Zheng, B.; Chi, X. D.; Ji, X F.; Huang, F. H. Chem. Commun. 2011, 47, 9840.

[15] Yang, W.; Li, Y. J.; Zhang, J. H.; Yu, Y. W.; Liu, T. F.; Liu, H. B.; Li, Y. L. Org. Biomol. Chem. 2011, 9, 6022.

[16] Yang, W.; Li, Y. J.; Zhang, J. H.; Chen, N.; Chen, S. H.; Liu, H. B.; Li, Y. L. J. Org. Chem. 2011, 76, 7750.

[17] Li, H.; Zhang, H.; Zhang, Q.; Zhang, Q. W.; Qu, D. H. Org. Lett. 2012, 14, 5900 .

[18] Zhang, H.; Zhou, B.; Li, H.; Qu, D. H.; Tian, H. J. Org. Chem. 2013, 78, 2091.

[19] Jiang, W.; Han, M.; Zhang, H. Y.; Zhang, Z. J.; Liu, Y. Chem. Eur. J. 2009, 15, 9938.

[20] Jiang, Q.; Zhang, H. Y.; Han, M.; Ding, Z. J.; Liu, Y. Org. Lett. 2010, 12, 1728.

[21] Li, S. J.; Weng, G. H.; Zheng, B.; Yan, X. Z.; Wu, J.; Lin, W.; Chen, H. X.; Zhang, X. C. Eur. J. Org. Chem. 2012, 33, 6570.

[22] Bissell, R. A.; Cordova, E.; Kaifer, A. E.; Stoddart, J. F. Nature 1994, 369, 133.

[23] Leigh, D. A.; Thomson, A. R. Org. Lett. 2006, 8, 5377.

[24] Yen, M.; Li, W. S.; Lai, C. C.; Chao, I.; Chiu, S. H. Org. Lett. 2006, 8,3223 .

[25] Zhou, W.; Li, J.; He, X.; Li, C.; Lv, J.; Li, Y.; Wang, S.; Liu, H.; Zhu, D. Chem. Eur. J. 2008, 14, 754

[26] Zheng, H. Y.; Zhou, W. D.; Lv, J.; Yin, X. D.; Li, Y. J.; Liu, H. B.; Li, Y. L. Chem. Eur. J. 2009, 15, 13253.

[27] Zhao, Y. J.; Li, Y. J.; Lai, S. W.; Yang, J.; Liu, C.; Liu, H. B.; Che, C. M.; Li, Y. L. Org. Biomol. Chem. 2011, 9, 7500.

[28] Huang, F. H.; Switek, K. A.; Gibson, H. W. Chem. Commun. 2005, 9,3655 .

[29] Zhang, Z. J.; Zhang, Y. M.; Liu, Y. J. Org. Chem. 2011, 76, 4682.

[30] iang, Y.; Guo, J.; Chen, C. Org. Lett. 2010, 12, 4248.

[31] Hu, S. Z.; Chen, C. F. Chem. Eur. J. 2011, 17, 5424.

[32] Khashab, N. M.; Trabolsi, A.; Lau, Y. A.; Ambrogio, M. W.; Friedman, D. C.; Khatib, H. A.; Zink, J. I.; Stoddart, J. F. Eur. J. Org. Chem. 2009, 11, 1669

[33] Khashab, N. M.; Belowich, M. E.; Trabolsi, A.; Friedman, D. C.; Valente, C.; Lau, Y.; Khatib, H. A.; Zink, J. I.; Stoddart, J. F. Chem. Commun. 2009, 36, 5371.

[34] Angelos, S.; Khashab, N. M.; Yang, Y. W.; Trabolsi, A.; Khatib, H. A.; Stoddart, J. F.; Zink, J. I. J. Am. Chem. Soc. 2009, 131, 12912.

[35] Thomas, C. R.; Ferris, D. P.; Lee, J. H.; Choi, E.; Cho, M. H.; Kim, E. S.; Stoddart, J. F.; Shin, J. S.; Cheon, J.; Zink, J. I. J. Am. Chem. Soc. 2009, 132, 10623 .

[36] Zhao, Y. L.; Li, Z.; Kabehie, S.; Botros, Y. Y.; Stoddart, J. F.; Zink, 
J. I. J. Am. Chem. Soc. 2009, 132, 13016.

[37] Meng, H.; Xue, M.; Xia, T.; Zhao, Y. L.; Tamanoi, F.; Stoddart, J. F.; Zink, J. I.; Nel, A. E. J. Am. Chem. Soc. 2010, 132, 12690.

[38] Boyle, M. M.; Smaldone, R. A.; Whalley, A. C.; Ambrogio, M. W.; Botros, Y. Y.; Stoddart, J. F. Chem. Sci. 2011, 2, 204.

[39] Ambrogio, M. W.; Thomas, C. R.; Zhao, Y. L.; Zink, J. I.; Stoddart, J. F. Acc. Chem. Res. 2011, 44, 903.

[40] (a) Yan, X.; Wang, F.; Zheng, B.; Huang, F. Chem. Soc. Rev. 2012 , $41,6042$.

(b) Yan, X.; Li, S.; Pollock, B. J.; Cook, T. R.; Chen, J.; Zhang, Y.; Ji, X.; Yu, Y.; Huang, F.; Stang, P. J. Proc. Natl. Acad. Sci. U. S. A. 2013, 110, 15585 . (c) Li, S.; Weng, G.-H.; Lin, W.; Sun, Z.-B.; Zhou, M.; Zhu, B.; Ye, Y.; Wu, J. Polym. Chem. 2014, 5, 3994.

(d) Wei, P.; Li, S.; Zhang, Y.; Yu, Y.; Yan, X. Polym. Chem. 2014, 5, 3972.

[41] Fahrenbach, A. C.; Warren, S. C.; Incorvati, J. T.; Avestro, A. J.; Barnes, J. C.; Stoddart, J. F.; Grzybowski, B. A. Adv. Mater. 2013, $25,331$.

[42] Wang, F.; Zhang, J. Q.; Ding, X.; Dong, S. Y.; Liu, M.; Zheng, B.; Li, S. J.; Wu, L.; Yu, Y. H.; Gibson, H. W.; Huang, F. H. Angew. Chem., Int. Ed. 2010, 49, 1090.

[43] Ji, X. F.; Yao, Y.; Li, J. Y.; Yan, X. Z.; Huang, F. H. J. Am. Chem. Soc. 2013, 135, 74 . 